\title{
Modeling and Dynamic Analysis in a Hybrid Stochastic Bioeconomic System with Double Time Delays and Lévy Jumps
}

\author{
Chao Liu $\mathbb{D}^{1,2,3}$ Longfei Yu, ${ }^{1,2}$ and Luping Wang ${ }^{1,2}$ \\ ${ }^{1}$ Institute of Systems Science, Northeastern University, Shenyang, China \\ ${ }^{2}$ State Key Laboratory of Integrated Automation of Process Industry, Northeastern University, Shenyang, China \\ ${ }^{3}$ Department of Applied Mathematics, Hong Kong Polytechnic University, Hung Hom, Hong Kong
}

Correspondence should be addressed to Chao Liu; singularsystem@163.com

Received 17 December 2017; Revised 30 May 2018; Accepted 12 July 2018; Published 13 August 2018

Academic Editor: Philippe Bogaerts

Copyright () 2018 Chao Liu et al. This is an open access article distributed under the Creative Commons Attribution License, which permits unrestricted use, distribution, and reproduction in any medium, provided the original work is properly cited.

\begin{abstract}
A double delayed hybrid stochastic prey-predator bioeconomic system with Lévy jumps is established and analyzed, where commercial harvesting on prey and environmental stochasticity on population dynamics are considered. Two discrete time delays are utilized to represent the maturation delay of prey and gestation delay of predator, respectively. For a deterministic system, positivity of solutions and uniform persistence of system are discussed. Some sufficient conditions associated with double time delays are derived to discuss asymptotic stability of interior equilibrium. For a stochastic system, existence and uniqueness of a global positive solution are studied. By using the invariant measure theory and singular boundary theory of diffusion process, existence of stochastic Hopf bifurcation and stochastic stability are investigated. By constructing appropriate Lyapunov functions, asymptotic dynamic behavior of the proposed hybrid stochastic system with double time delays and Lévy jumps is discussed. Numerical simulations are provided to show consistency with theoretical analysis.
\end{abstract}

\section{Introduction}

It is well known that a harvest effort has a strong dynamical impact on the prey-predator system [1-5], which plays a significant role in bioeconomics management among various species in a harvested prey-predator system [6-8]. Furthermore, it is more realistic to investigate the coexistence and interaction mechanism of the harvested prey-predator system by introducing time delays into a model system, such as maturation delay and gestation delay of the population [9-12]. In recent years, combined dynamic effects of the harvest effort and time delay on the population dynamics of prey-predator systems have been widely investigated in [13-18] and references therein, where the asymptotic behavior of the model system around equilibrium is analyzed and stability of bifurcated periodic solutions is studied. Yuan et al. [18] incorporated gestation delay $\tau>0$ as a negative feedback of predator population density in the harvested prey-predator system, which is as follows:

$$
\begin{aligned}
& \dot{x}_{1}(t)=x_{1}(t)\left(r_{1}-b_{1} x_{1}(t)\right)-\frac{a_{1} x_{1}(t) x_{2}(t)}{k+x_{1}(t)}-\frac{q E x_{1}(t)}{m_{1} E+m_{2} x_{1}(t)}, \\
& \dot{x}_{2}(t)=x_{2}(t)\left(r_{2}-\frac{a_{2} x_{2}(t-\tau)}{k+x_{1}(t-\tau)}\right),
\end{aligned}
$$

where $x_{1}(t)$ and $x_{2}(t)$ represent the population density of the prey and predator population, respectively. $r_{1}$ and $r_{2}$ stand for the birth rate of the prey and predator population, respectively. $b_{1}$ represents the intracompetition rate for the 
prey population, $a_{1}$ is the maximum value of the per capita reduction rate of the prey population due to predation, and $a_{2}$ has a similar interpretation to that of $a_{1} . k$ measures the extent to which the surrounding environment provides protection to each population. $q$ denotes the catchability coefficient, $E$ is a constant parameter representing the harvest effort on the prey population, and $\tau>0$ represents the gestation delay of the predator population. By analyzing the dynamical behavior of interior equilibrium and properties of bifurcation phenomena, it reveals that sustainable development of the harvested prey-predator system may be guaranteed by adopting an appropriate harvest effort.

It should be noted that the dynamical behavior of the commercially harvested bioeconomic system can be precisely predicted by using stochastic mathematical models [19-23], which can provide an additional degree of realistic reflection in the real world compared to its corresponding deterministic counterpart. Many scholars have incorporated stochastic perturbations into deterministic mathematical models to discuss dynamic effects of environmental noises on population dynamics of the harvested bioeconomic system [19-21], which show that persistence and extinction of population are relevant to time delay and stochastic fluctuations. Combined dynamic effects of time delay and Gaussian white noises on population dynamics of the harvested bioeconomic system as well as optimal harvest control problems are studied in [22, 23]. Recently, it is proved that Lévy jumps can efficiently depict sudden and severe environmental perturbations arising in the real world $[24,25]$, while these phenomena cannot be described better by Brownian motion.

Based on the above analysis, some assumptions are proposed as follows.

Assumption 1. In this paper, we will extend the work in [18] by incorporating commercial harvesting on prey into system (1). $E(t)$ represents the commercial harvesting effort on prey at time $t, w$ represents the harvesting reward coefficients, $c$ represents the cost per unit harvesting effort for the unit weight of prey, and $v$ is the economic interest of commercial harvesting on prey. Based on the economic theory proposed in [26], an algebraic equation is constructed to study the economic interest of commercial harvesting:

Net economic revenue $=$ total revenue $(\mathrm{TR})-$ total cost $(\mathrm{TC})$.

Based on system (1), TR and TC in (2), it is easy to show that $\mathrm{TR}=\mathrm{wE}(t) x_{1}(t)$ and $\mathrm{TC}=c E(t)$.

Assumption 2. In this paper, the maturity of the prey population is assumed to be mediated by discrete time delay $\tau_{1}>0$. Furthermore, the reproduction of the predator population after predating the prey population is not instantaneous but will be mediated by some time lag required for gestation of the predator population. $\tau_{2}>0$ represents the gestation delay of the predator population. Hence, we will extend the work in [18] by incorporating two different discrete time delays into system (1), and $\tau_{1} \neq \tau_{2}$.

Assumption 3. In this paper, the population growth of prey and predator populations affected by environmental stochastic fluctuations is assumed to be a stochastic process. Gaussian white noises and Lévy jumps will be incorporated into system (1) to describe stochastic surrounding environmental factors. $\sigma_{j k}(j, k=1,2)$ are nonnegative constants, $\xi_{1}(t)$ and $\xi_{2}(t)$ denote multiplicative stochastic excitation and external stochastic excitation related to surrounding environment, respectively. $\xi_{j}(t)(j=$ $1,2)$ represents independent Gaussian white noise such that $\mathbb{E}\left[\xi_{j}(t)\right]=0(j=1,2)$. For any time, $t_{1} \neq t_{2}, \mathbb{E}\left[\xi_{j}\left(t_{1}\right) \xi_{j}\left(t_{2}\right)\right]=$ $\delta\left(t_{2}-t_{1}\right)(j=1,2)$, and $\mathbb{E}\left[\xi_{j}\left(t_{1}\right) \xi_{k}\left(t_{2}\right)\right]=0(j, k=1,2, j \neq k)$, where $\delta$ denotes the Dirac delta function. $x_{i}(t-)$ represents the left limit of $x_{i}(t), i=1,2$ and $\gamma_{i}(u)>-1, i=1,2$. $N$ denotes a Poisson counting measure with characteristic measure $\lambda$ on a measurable subset $\mathbb{Y}$ with $\lambda(\mathbb{Y})<+\infty$ and $\lambda$ is assumed to be a Lévy measure such that $\tilde{N}(\mathrm{~d} t, \mathrm{~d} u)=$ $N(\mathrm{~d} t, \mathrm{~d} u)-\lambda(\mathrm{d} u) \mathrm{d} t$ and $\mathbb{Y}$ denotes a measurable subset of $\mathbb{R}_{+}$. Throughout this paper, $\xi_{i}(t)(i=1,2)$ and $N$ are assumed to be independent.

In this paper, keeping all these Assumptions 1-3 in mind, a double delayed hybrid stochastic prey-predator bioeconomic system with Lévy jumps is established as follows:

$$
\begin{aligned}
\mathrm{d} x_{1}(t)= & {\left[x_{1}\left(t-\tau_{1}\right)\left(r_{1}-b_{1} x_{1}\left(t-\tau_{1}\right)\right)-\frac{a_{1} x_{1}(t) x_{2}(t)}{k+x_{1}(t)}\right.} \\
& \left.-\frac{q E(t) x_{1}(t)}{m_{1} E(t)+m_{2} x_{1}(t)}\right] \mathrm{d} t \\
& +\left[\sigma_{11} x_{1}(t) \xi_{1}(t)+\sigma_{12} \xi_{2}(t)\right] \mathrm{d} t \\
& +\int_{\mathbb{Y}} \gamma_{1}(u) x_{1}(t-) \tilde{N}(\mathrm{~d} t, \mathrm{~d} u), \\
\mathrm{d} x_{2}(t)= & x_{2}(t)\left(r_{2}-\frac{a_{2} x_{2}\left(t-\tau_{2}\right)}{k+x_{1}\left(t-\tau_{2}\right)}\right) \mathrm{d} t \\
& +\left[\sigma_{21} x_{2}(t) \xi_{1}(t)+\sigma_{22} \xi_{2}(t)\right] \mathrm{d} t \\
& +\int_{\mathbb{Y}} \gamma_{2}(u) x_{2}(t-) \tilde{N}(\mathrm{~d} t, \mathrm{~d} u), \\
0= & E(t)\left(w x_{1}(t)-c\right)-v,
\end{aligned}
$$

where the initial conditions for system (3) take the following form:

$$
\begin{gathered}
x_{1}(\theta) \geq 0, \\
x_{2}(\theta) \geq 0, \\
\theta \in\left[-\max \left\{\tau_{1}, \tau_{2}\right\}, 0\right], \\
E(0) \geq 0 .
\end{gathered}
$$


System (3) can be rewritten in the matrix form as follows:

$$
\begin{aligned}
\Xi(t)\left[\begin{array}{c}
\mathrm{d} x_{1}(t) \\
\mathrm{d} x_{2}(t) \\
0
\end{array}\right] & =\left[\begin{array}{c}
F_{1}\left(x_{1}, x_{2}, E\right) \\
F_{2}\left(x_{1}, x_{2}, E\right) \\
F_{3}\left(x_{1}, x_{2}, E\right)
\end{array}\right] \\
& =\left[\begin{array}{c}
{\left[x_{1}\left(t-\tau_{1}\right)\left(r_{1}-b_{1} x_{1}\left(t-\tau_{1}\right)\right)-\frac{a_{1} x_{1}(t) x_{2}(t)}{k+x_{1}(t)}-\frac{q E(t) x_{1}(t)}{m_{1} E(t)+m_{2} x_{1}(t)}\right] \mathrm{d} t+\left[\sigma_{11} x_{1}(t) \xi_{1}(t)+\sigma_{12} \xi_{2}(t)\right] \mathrm{d} t+\int_{\curlyvee} \gamma_{1}(u) x_{1}(t-) \tilde{N}(\mathrm{~d} t, \mathrm{~d} u)} \\
x_{2}(t)\left(r_{2}-\frac{a_{2} x_{2}\left(t-\tau_{2}\right)}{k+x_{1}\left(t-\tau_{2}\right)}\right) \mathrm{d} t+\left[\sigma_{21} x_{2}(t) \xi_{1}(t)+\sigma_{22} \xi_{2}(t)\right] \mathrm{d} t+\int_{\curlyvee} \gamma_{2}(u) x_{2}(t-) \tilde{N}(\mathrm{~d} t, \mathrm{~d} u) \\
E(t)\left(w x_{1}(t)-c\right)-v
\end{array}\right]
\end{aligned}
$$

Remark 1. Since the algebraic equation in (3) includes no differentiated variables, the third row in matrix

$$
\Xi(t)=\left[\begin{array}{lll}
1 & 0 & 0 \\
0 & 1 & 0 \\
0 & 0 & 0
\end{array}\right]
$$

has a corresponding zero row.

Recently, some hybrid bioeconomic systems with time delay and stochastic fluctuations are established in [27-30] to investigate the combined dynamic effects of stochastic fluctuation and commercial harvesting on population dynamics. These proposed systems in [27-30] are also constructed by several differential equations with stochastic fluctuations and an algebraic equation. Compared with the previously bioeconomic systems proposed in [19-23, 31] and the references therein, such delayed bioeconomic systems [27-30] can not only discuss coexistence and interaction mechanism of a delayed bioeconomic system under stochastic environmental fluctuations but also investigate population dynamics due to variations of economic interest of commercial harvesting. However, biological characteristics among interacting populations are not considered in [27-29]; time delays such as gestation delay and maturation delay for interacting populations in [27-29] are assumed to be the same discrete value, which contradicts to reality in the real world. Asymptotical stability of interior equilibrium and dynamic effects of Lévy jumps on population dynamics are not discussed in [27-29]. It is proved that Lévy jumps can efficiently depict sudden and severe environmental perturbations arising in the real world [24, 25], while these phenomena can not be described better by Brownian motion. Although the dynamic effects of double time delays have been investigated in [30], the combined dynamic effects of multiple time delays and Lévy jumps on population dynamics have not been investigated in [30]. Dynamic effects of multiple time delays on the hybrid bioeconomic prey-predator system are investigated in $[15,32]$. However, the dynamic effect of Lévy jumps and asymptotical stability of solutions of the stochastic system are not studied in $[15,32]$.

To the authors' best knowledge, population dynamics of the hybrid bioeconomic system with double time delays and Lévy jumps have not been investigated. The rest sections of this paper are organized as follows: in the second section, qualitative analysis of the deterministic system is investigated. Positivity of solutions and uniform persistence of the deterministic system are studied. Asymptotic stability of interior equilibrium of the deterministic system is discussed due to variations of double time delays. In the third section, qualitative analysis of the stochastic system is investigated. Existence and uniqueness of global positive solution are studied. In the absence of double time delays and Lévy jumps, the existence of stochastic Hopf bifurcation and stochastic stability is investigated. In the presence of double time delays and Lévy jumps, by constructing appropriate Lyapunov functions, the asymptotic dynamic behavior of the proposed hybrid stochastic system with double time delays and Lévy jumps is discussed. In the fourth section, numerical simulations are provided to support theoretical findings. Finally, this paper ends with a conclusion.

\section{Qualitative Analysis of Deterministic System}

In the absence of stochastic fluctuations, positivity of solutions and uniform persistence of system (3) with initial conditions (4) will be studied in Lemmas 1 and 2.

Lemma 1. When $\sigma_{j k}=0(j, k=1,2)$ and $\gamma_{i}(u)=0(i=1,2)$, all solutions of system (3) with initial conditions (4) are positive for all $t \geq 0$.

Proof 1 . When $\sigma_{j k}=0(j, k=1,2)$ and $\gamma_{i}(u)=0(i=1,2)$, it is easy to show that $F_{i}: \mathbb{R}_{+}^{3+1} \rightarrow \mathbb{R}^{3}$ is locally Lipschitz and satisfy the condition, $F_{i}>0$, where $F_{i}(i=1,2,3)$ can be found in (5). 
Due to lemma in [33] and Theorem A.4 in [34], all solutions of system (3) with initial conditions (4) exist uniquely and each component of the solution remains within the interval $\left[0, U_{0}\right)$ for some $U_{0}>0$. Standard and simple arguments show that any solution of system (3) with initial conditions (4) always exist and stay positive.

Lemma 2. When $\sigma_{j k}=0(j, k=1,2)$ and $\gamma_{i}(u)=0(i=1,2)$, if $v>0$ and $\tau_{1}$ are bounded and satisfy the following inequality:

$$
\tau_{1}<\min \left\{\frac{1}{2 r_{1}} \ln \frac{c b_{1}}{w r_{1}}, \frac{1}{2 r_{1}} \ln \frac{\sqrt{\left[b_{1} c k\left(m r_{1}+q\right)\right]^{2}+c w k m_{1}^{2} r_{1}^{4}}-b_{1} c k\left(m r_{1}+q\right)}{8 c m_{1} r_{1}^{2}}\right\}
$$

then all solutions of system (3) with initial conditions (4) are uniformly persistent.

Proof 2. When $\sigma_{j k}=0(j, k=1,2)$ and $\gamma_{i}(u)=0(i=1,2)$, by using Taylor series expansion [35], for $x_{1}(t), x_{2}(t), \tau_{1}>0$, and $\tau_{2}>0$, it is easy to show that

$$
\begin{aligned}
& x_{1}\left(t-\tau_{1}\right)=x_{1}(t)-\tau_{1} \frac{\mathrm{d}}{\mathrm{d} t}\left(x_{1}(t)-\tau_{1} \dot{x}_{1}(t)+\cdots\right), \\
& x_{2}\left(t-\tau_{2}\right)=x_{2}(t)-\tau_{2} \frac{\mathrm{d}}{\mathrm{d} t}\left(x_{2}(t)-\tau_{2} \dot{x}_{2}(t)+\cdots\right) .
\end{aligned}
$$

Hence, it follows that there exists $T_{1}>0$ such that

$$
\begin{aligned}
& x_{1}\left(t-\tau_{1}\right) \leq x_{1}(t), \\
& x_{2}\left(t-\tau_{2}\right) \leq x_{2}(t) .
\end{aligned}
$$

According to Lemma 1, (9), and the first equation of system (3), it follows from simple computations that $\dot{x}_{1}(t)<r_{1} x_{1}\left(t-\tau_{1}\right) \leq r_{1} x_{1}(t)$, which derives that

$$
x_{1}(t) \leq x_{1}\left(t-\tau_{1}\right) e^{r_{1} \tau_{1}}
$$

holds for $t>T_{2}:=T_{1}+\tau_{1}$.

For $t>T_{2}$, it follows from (10) and the first equation of system (3) that

$$
\dot{x}_{1}(t)<x_{1}(t)\left[r_{1}-b_{1} x_{1}\left(t-\tau_{1}\right)\right]<x_{1}(t)\left[r_{1}-b_{1} x_{1}(t) e^{-2 r_{1} \tau_{1}}\right],
$$

which gives that

$$
\limsup _{t \rightarrow+\infty} x_{1}(t) \leq \frac{r_{1} e^{2 r_{1} \tau_{1}}}{b_{1}}:=P_{1} .
$$

If $\tau_{1}$ is bounded, then $P_{1}$ is bounded for $t>T_{2}$.

Based on Lemma 1 and the second equation of system (3), there exists $T_{3}>T_{2}$ and it follows from simple computations that $\dot{x}_{2}(t)>r_{2} x_{2}(t)\left(1-\left(a_{2} x_{2}(t) / k r_{2}\right)\right)$ holds for $t>T_{3}$. By using standard comparison arguments, it gives that

$$
\liminf _{t \rightarrow+\infty} x_{2}(t) \geq \frac{k r_{2}}{a_{2}}:=Q_{2} .
$$

According to biological interpretations, (12) and the first equation of system (3), it derives that there exists $T_{4}>T_{3}$ and $r_{1} x_{1}(t)-b_{1} e^{-2 r_{1} \tau_{1}} x_{1}^{2}(t)-\left(\left(a_{1} x_{1}(t) x_{2}(t)\right) /(k+\right.$ $\left.\left.\left(r_{1} / b_{1}\right) e^{2 r_{1} \tau_{1}}\right)\right)>0$ holds for $t>T_{4}$. Furthermore, it follows from Lemma 1 that $a_{1} x_{2}(t)<r_{1}\left(k+\left(r_{1} / b_{1}\right) e^{2 r_{1} \tau_{1}}\right)$ and

$$
\limsup _{t \rightarrow+\infty} x_{2}(t) \leq \frac{r_{1}}{a_{1}}\left(k+\frac{r_{1}}{b_{1}} e^{2 r_{1} \tau_{1}}\right):=P_{2}
$$

If $\tau_{1}$ is bounded, then $P_{2}$ is bounded for $t>T_{4}$.

By using the first equation of system (3) and (14), it can be obtained that there exists $T_{5}>T_{4}$ such that

$$
\dot{x}_{1}(t)>r_{1} e^{-r_{1} \tau_{1}} x_{1}(t)-b_{1} x_{1}^{2}(t)-\frac{a_{1} P_{2} x_{1}(t)}{k}-\frac{q x_{1}(t)}{m_{1}}
$$

holds for $t>T_{5}$ and

$$
\liminf _{t \rightarrow+\infty} x_{1}(t) \geq \frac{m_{1} k r_{1}^{2} e^{-2 r_{1} \tau_{1}}}{4 b_{1}\left(a_{1} m_{1} P_{2}+q k\right)}:=Q_{1}
$$

Furthermore, by using simple computations, it follows from (12) and (16) and the third algebraic equation of system (3) that

$$
\begin{aligned}
& \lim \inf _{t \rightarrow+\infty} E(t) \geq \frac{v}{w P_{1}-c}:=Q_{3}, \\
& \lim \sup _{t \rightarrow+\infty} E(t) \leq \frac{v}{w Q_{1}-c}:=P_{3} .
\end{aligned}
$$

If $\tau_{1}<\left(1 / 2 r_{1}\right) \ln \left(c b_{1} / w r_{1}\right)$, then it is easy to show that $Q_{3}>0$ and $Q_{3}$ is bounded.

If $\tau_{1}<\left(1 / 2 r_{1}\right) \ln \left(\sqrt{\left[b_{1} c k\left(m r_{1}+q\right)\right]^{2}+c w k m_{1}^{2} r_{1}^{4}}-b_{1} c k\right.$ $\left.\left(m r_{1}+q\right) / 8 c m_{1} r_{1}^{2}\right)$, then it is easy to show that $P_{3}>0$ and $P_{3}$ are bounded.

Based on the above analysis, if $v>0$ and $\tau_{1}$ are bounded and satisfy the following inequality 


$$
\tau_{1}<\min \left\{\frac{1}{2 r_{1}} \ln \frac{c b_{1}}{w r_{1}}, \frac{1}{2 r_{1}} \ln \frac{\sqrt{\left[b_{1} c k\left(m r_{1}+q\right)\right]^{2}+c w k m_{1}^{2} r_{1}^{4}}-b_{1} c k\left(m r_{1}+q\right)}{8 c m_{1} r_{1}^{2}}\right\}
$$

then all solutions of system (3) with initial conditions (4) are uniformly persistent.

Remark 2. From the practical and physical perspective of viewpoints, positivity of the solution of the proposed hybrid delayed stochastic bioeconomic system refers to each population survival for a long duration under combined dynamic effects of commercial harvesting, time delay, and stochastic fluctuations. Since natural resources for each population survival are relatively limited within a closed environment, permanence of the hybrid delayed stochastic bioeconomic system interprets that there exist positive finite upper bounds and lower bounds for each population density, which may avoid overpopulation and extinction of each interacting populations. Furthermore, in order to maintain sustainable development of commercially harvested population, commercial harvesting should be constrained within a certain range. It practically interprets there exist positive finite upper bounds and lower bounds for commercial harvesting amount on the predator population.

When economic interest $v>0, \sigma_{j k}=0(j, k=1,2)$, and $\gamma_{i}(u)=0(i=1,2)$, the interior equilibrium of system (3) can be obtained as follows: $M^{*}\left(x_{1}^{*}, x_{2}^{*}, E^{*}\right)=\left(x_{1}^{*},\left(r_{2} k / a_{2}-\right.\right.$ $\left.\left.r_{2}\right),\left(v / w x_{1}^{*}-c\right)\right)$ and $x_{1}^{*}$ satisfies the following equation:

$$
x_{1}^{* 4}+A_{1} x_{1}^{* 3}+A_{2} x_{1}^{* 2}+A_{3} x_{1}^{*}+A_{4}=0,
$$

where $A_{i}, i=1,2,3,4$ are defined as follows:

$$
\begin{aligned}
& A_{1}=\frac{m_{2}\left(a_{2}-r_{2}\right)\left[w\left(b_{1} k-r_{1}\right)-c b_{1}\right]}{w m_{2} b_{1}\left(a_{2}-r_{2}\right)}, \\
& A_{2}=\frac{w m_{2} k\left[a_{1} r_{2}-r_{1}\left(a_{2}-r_{2}\right)\right]+\left(a_{2}-r_{2}\right)\left[b_{1} m_{1} v-c m_{2}\left(b_{1} k-r_{1}\right)\right]}{w m_{2} b_{1}\left(a_{2}-r_{2}\right)}, \\
& A_{3}=\frac{c m_{2}\left[k r_{1}\left(a_{2}-r_{2}\right)-a_{1} r_{2} k\right]+v\left(a_{2}-r_{2}\right)\left[q+m_{1}\left(b_{1} k-r_{1}\right)\right]}{w m_{2} b_{1}\left(a_{2}-r_{2}\right)}, \\
& A_{4}=\frac{a_{1} r_{2} m_{1} v-q\left(a_{2}-r_{2}\right)\left(q+m_{1} r_{1}\right)}{w m_{2} b_{1}\left(a_{2}-r_{2}\right)} .
\end{aligned}
$$

According to Routh-Hurwitz criterion [35], a sufficient condition for (19) has at least one positive root which is $A_{4}<0$. Furthermore, $M^{*}$ exists provided that $x_{2}^{*}=\left(r_{2} k / a_{2}-r_{2}\right)>0$ and $E^{*}=\left(v / w x_{1}^{*}-c\right)>0$. Based on the above analysis, if $\left(\tau_{1}, \tau_{2}, v\right) \in H_{1}$, then there exists at least one positive root for (19), and $H_{1}$ is defined as follows:

$$
H_{1}=\left\{\left(\tau_{1}, \tau_{2}, v\right) \mid \tau_{1} \geq 0, \tau_{2} \geq 0,0<v<\min \left\{\frac{q\left(a_{2}-r_{2}\right)\left(q+m_{1} r_{1}\right)}{a_{1} r_{2} m_{1}}, \frac{1}{2 r_{1}} \ln \frac{c b_{1}}{w r_{1}}, \frac{1}{2 r_{1}} \ln \frac{\sqrt{\left[b_{1} c k\left(m r_{1}+q\right)\right]^{2}+c w k m_{1}^{2} r_{1}^{4}}-b_{1} c k\left(m r_{1}+q\right)}{8 c m_{1} r_{1}^{2}}\right\}\right\} .
$$

In the following part, some sufficient conditions associated with double time delays are derived to investigate the local asymptotic stability of the system (3) around $M^{*}$. By using the third algebraic equation $E(t)=v /\left(w x_{1}(t)-c\right)$, the system (3) can be rewritten as follows:

$$
\begin{aligned}
\dot{x}_{1}(t)= & x_{1}\left(t-\tau_{1}\right)\left[r_{1}-b_{1} x_{1}\left(t-\tau_{1}\right)\right]-\frac{a_{1} x_{1}(t) x_{2}(t)}{k+x_{1}(t)} \\
& -\frac{q v x_{1}(t)}{m_{1} v+m_{2} x_{1}(t)\left(w x_{1}(t)-c\right)}, \\
\dot{x}_{2}(t)= & x_{2}(t)\left[r_{2}-\frac{a_{2} x_{2}\left(t-\tau_{2}\right)}{k+x_{1}\left(t-\tau_{2}\right)}\right] .
\end{aligned}
$$

For mathematical convenience, some transformations $x_{1}(t)=x_{1}^{*} e^{y_{1}(t)}$ and $x_{2}(t)=x_{2}^{*} e^{y_{2}(t)}$ are made and system (22) is rewritten as follows:

$$
\begin{aligned}
\dot{y}_{1}(t)= & -\frac{b_{1} x_{1}^{*} e^{y_{1}\left(t-\tau_{1}\right)}}{e^{y_{1}(t)}}\left(e^{y_{1}\left(t-\tau_{1}\right)}-1\right) \\
& +a_{1} x_{2}^{*}\left(\frac{e^{y_{1}\left(t-\tau_{1}\right)}}{e^{y_{1}(t)}} \frac{1}{k+x_{1}^{*}}-\frac{e^{y_{2}(t)}}{k+x_{1}(t)}\right) \\
& +q v\left[\frac{e^{y_{1}\left(t-\tau_{1}\right)}}{e^{y_{1}(t)}} \frac{1}{m_{1} v+m_{2} x_{1}^{*}\left(w x_{1}^{*}-c\right)}\right. \\
& \left.\quad-\frac{1}{m_{1} v+m_{2} x_{1}(t)\left(w x_{1}(t)-c\right)}\right], \\
\dot{y}_{2}(t)= & \frac{a_{2} x_{2}^{*}\left[x_{1}^{*}\left(e^{y_{1}\left(t-\tau_{2}\right)}-1\right)-\left(k+x_{1}^{*}\right)\left(e^{y_{2}\left(t-\tau_{2}\right)}-1\right)\right]}{\left(k+x_{1}^{*}\right)\left(k+x_{1}^{*} e^{y_{1}\left(t-\tau_{2}\right)}\right)} .
\end{aligned}
$$

By utilizing the following mathematical relations: 


$$
\begin{aligned}
& e^{y_{1}\left(t-\tau_{1}\right)}=e^{y_{1}(t)}-\int_{t-\tau_{1}}^{t} e^{y_{1}(s)} \frac{\mathrm{d} y_{1}}{\mathrm{~d} s} \mathrm{~d} s, \\
& e^{y_{1}\left(t-\tau_{2}\right)}=e^{y_{1}(t)}-\int_{t-\tau_{2}}^{t} e^{y_{1}(s)} \frac{\mathrm{d} y_{1}}{\mathrm{~d} s} \mathrm{~d} s,
\end{aligned}
$$

$$
\begin{aligned}
& e^{y_{2}\left(t-\tau_{1}\right)}=e^{y_{2}(t)}-\int_{t-\tau_{1}}^{t} e^{y_{2}(s)} \frac{\mathrm{d} y_{2}}{\mathrm{~d} s} \mathrm{~d} s, \\
& e^{y_{2}\left(t-\tau_{2}\right)}=e^{y_{2}(t)}-\int_{t-\tau_{2}}^{t} e^{y_{2}(s)} \frac{\mathrm{d} y_{2}}{\mathrm{~d} s} \mathrm{~d} s,
\end{aligned}
$$

system (23) can be rewritten as follows:

$$
\begin{aligned}
\dot{y}_{1}(t)= & -\frac{b_{1} x_{1}^{*} e^{y_{1}\left(t-\tau_{1}\right)}\left(e^{y_{1}(t)}-1\right)}{e^{y_{1}(t)}}-\frac{a_{1} k x_{2}^{*}\left(e^{y_{2}(t)}-1\right)}{\left(k+x_{1}(t)\right)\left(k+x_{1}^{*}\right)}+\frac{q v m_{2} x_{1}^{*}\left(w x_{1}^{*}-c\right)\left(e^{y_{1}(t)}-1\right)}{\left[m_{1} v+m_{2} x_{1}^{*}\left(w x_{1}^{*}-c\right)\right]\left[m_{1} v+m_{2} x_{1}(t)\left(w x_{1}^{*}-c\right)\right]} \\
& +\left(\frac{b_{1} x_{1}^{*} e^{y_{1}\left(t-\tau_{1}\right)}-a_{1} x_{2}^{*}}{e^{y_{1}(t)}}-\frac{q v}{e^{y_{1}(t)}\left[m_{1} v+m_{2} x_{1}^{*}\left(w x_{1}^{*}-c\right)\right]}\right) \int_{t-\tau_{1}}^{t} e^{y_{1}(s)} \frac{\mathrm{d} y_{1}}{\mathrm{~d} s} \mathrm{~d} s, \\
\dot{y}_{2}(t)= & \frac{a_{2} x_{1}^{*} x_{2}^{*}\left(e^{y_{1}(t)}-1\right)}{\left(k+x_{1}^{*}\right)\left(k+x_{1}\left(t-\tau_{2}\right)\right)}-\frac{a_{2} x_{2}^{*}\left(e^{y_{2}(t)}-1\right)}{k+x_{1}\left(t-\tau_{2}\right)}-\frac{a_{2} x_{1}^{*} x_{2}^{*}}{\left(k+x_{1}^{*}\right)\left(k+x_{1}\left(t-\tau_{2}\right)\right)} \int_{t-\tau_{2}}^{t} e^{y_{1}(s)} \frac{\mathrm{d} y_{1}}{\mathrm{~d} s} \mathrm{~d} s+\frac{a_{2} x_{2}^{*}}{k+x_{1}\left(t-\tau_{2}\right)} \int_{t-\tau_{2}}^{t} e^{y_{2}(s)} \frac{\mathrm{d} y_{2}}{\mathrm{~d} s} \mathrm{~d} s .
\end{aligned}
$$

Some sufficient conditions for local asymptotical stability of system (3) around the interior equilibrium $M^{*}$ can be concluded as follows.

Theorem 1. When $\sigma_{j k}=0(j, k=1,2)$ and $\gamma_{i}(u)=0(i=1,2)$, if $\left(\tau_{1}, \tau_{2}, v\right) \in H_{1} \cap H_{2}$, then system (3) is asymptotically stable around interior equilibrium $M^{*}$, where $\mathrm{H}_{2}$ is defined in (A.10).

Proof 3. The proof of Theorem 1 can be found in Appendix A of this paper.

\section{Qualitative Analysis of Stochastic System}

If $\left(\tau_{1}, \tau_{2}, v\right) \in H_{1} \cap H_{2}$, then it follows from the third algebraic equation of system (3) that $E(t)=v /\left(w x_{1}(t)-c\right)$. Consequently, system (3) is transformed as follows:

$$
\begin{aligned}
\mathrm{d} x_{1}(t)= & {\left[x_{1}\left(t-\tau_{1}\right)\left(r_{1}-b_{1} x_{1}\left(t-\tau_{1}\right)\right)-\frac{a_{1} x_{1}(t) x_{2}(t)}{k+x_{1}(t)}\right.} \\
& \left.-\frac{q v x_{1}(t)}{m_{1} v+m_{2} x_{1}(t)\left(w x_{1}(t)-c\right)}\right] \mathrm{d} t \\
& +\left[\sigma_{11} x_{1}(t) \xi_{1}(t)+\sigma_{12} \xi_{2}(t)\right] \mathrm{d} t \\
& +\int_{\mathbb{Y}} \gamma_{1}(u) x_{1}(t-) \tilde{N}(\mathrm{~d} t, \mathrm{~d} u), \\
\mathrm{d} x_{2}(t)= & x_{2}(t)\left(r_{2}-\frac{a_{2} x_{2}\left(t-\tau_{2}\right)}{k+x_{1}\left(t-\tau_{2}\right)}\right) \mathrm{d} t \\
& +\left[\sigma_{21} x_{2}(t) \xi_{1}(t)+\sigma_{22} \xi_{2}(t)\right] \mathrm{d} t \\
& +\int_{\mathbb{Y}} \gamma_{2}(u) x_{2}(t-) \tilde{N}(\mathrm{~d} t, \mathrm{~d} u) .
\end{aligned}
$$

In the following part, existence and uniqueness of the global positive solution of system (26) are discussed in Theorem 2.
Theorem 2. If $\int_{\mathbb{Y}}\left[\gamma_{i}(u)-\ln \left(1+\gamma_{i}(u)\right)\right] \lambda \mathrm{d} u \leq \tilde{\gamma}_{i}(i=1,2)$ and $\tilde{\gamma}_{i}$ are positive constants and $\sigma_{j k}>0(j, k=1,2)$ is sufficiently small, then system (26) has a unique global positive solution for all $t>0$ and the solution $\left(x_{1}(t), x_{2}(t)\right) \in \mathbb{R}_{+}^{2}$ for $t>0$ almost surely.

Proof 4. The proof of Theorem 2 can be found in Appendix B of this paper.

3.1. Case I: System (3) without Double Time Delays and Lévy Jumps. In this subsection, when $\tau_{1}=\tau_{2}=0$ and $\gamma_{i}(u)=0$ $(i=1,2)$, it follows from simple computations that the linearized form of system (26) around $\left(x_{1}^{*}, x_{2}^{*}\right)$ is as follows:

$$
\begin{aligned}
& \dot{x}_{1}(t)=\alpha_{11} x_{1}(t)+\alpha_{12} x_{2}(t)+\sigma_{11} x_{1}(t) \xi_{1}(t)+\sigma_{12} \xi_{2}(t), \\
& \dot{x}_{2}(t)=\alpha_{21} x_{1}(t)+\alpha_{22} x_{2}(t)+\sigma_{21} x_{2}(t) \xi_{1}(t)+\sigma_{22} \xi_{2}(t),
\end{aligned}
$$

where $\alpha_{11}=r_{1}-2 b_{1} x_{1}^{*}-\left(a_{1} k x_{2}^{*} /\left(\left(k+x_{1}^{*}\right)^{2}\right)\right)-\left(q m_{1} v^{2} /\left(\left[m_{1} v+\right.\right.\right.$ $\left.\left.\left.\left(w_{1} x_{1}^{*}-c\right) m_{2} x_{1}^{*}\right]^{2}\right)\right), \quad \alpha_{12}=-\left(a_{1} x_{1}^{*} /\left(k+x_{1}^{*}\right)\right), \quad \alpha_{21}=a_{2} x_{2}^{* 2} /($ $\left.\left(k+x_{1}^{*}\right)^{2}\right)$, and $\alpha_{22}=r_{2}-\left(a_{2} x_{2}^{*} /\left(\left(k+x_{2}^{*}\right)^{2}\right)\right)$.

By using Khasminskii transformations, $x_{1}(t)=\omega \cos \theta$, $x_{2}(t)=\omega \sin \theta$, and system (27) are rewritten as follows:

$$
\begin{aligned}
\dot{\omega}(t)= & \omega\left[\alpha_{11} \cos ^{2} \theta+\left(\alpha_{11}+\alpha_{21}\right) \sin \theta \cos \theta+\alpha_{22} \sin ^{2} \theta\right] \\
& +\omega\left(\sigma_{11} \cos ^{2} \theta+\sigma_{21} \sin ^{2} \theta\right) \xi_{1}(t) \\
& +\left(\sigma_{12} \cos \theta+\sigma_{22} \sin \theta\right) \xi_{2}(t), \\
\dot{\theta}(t)= & \alpha_{21} \cos ^{2} \theta-\alpha_{12} \sin ^{2} \theta+\left(\alpha_{22}-\alpha_{11}\right) \cos \theta \sin \theta \\
& +\left(\sigma_{21}-\sigma_{11}\right) \sin \theta \cos \theta \xi_{1}(t) \\
& +\frac{\sigma_{22} \cos \theta-\sigma_{12} \sin \theta}{\omega} \xi_{2}(t) .
\end{aligned}
$$


According to Khasminskii limit theorem [36, 37], if $\tau_{1}=\tau_{2}=0, \quad \gamma_{i}(u)=0(i=1,2)$, and $\sigma_{j k}>0(j, k=1,2)$ are sufficiently small, then $\{\omega(t), \theta(t)\}$ weakly converges to the two-dimensional Markov diffusion process. Based on the stochastic averaging method, the Itô stochastic differential equation can be obtained as follows:

$$
\begin{aligned}
& \mathrm{d} \omega=f_{\omega} \mathrm{d} t+\beta_{11} \mathrm{~d} W_{\omega}+\beta_{12} \mathrm{~d} W_{\theta}, \\
& \mathrm{d} \theta=f_{\theta} \mathrm{d} t+\beta_{21} \mathrm{~d} W_{\omega}+\beta_{22} \mathrm{~d} W_{\theta},
\end{aligned}
$$

where $W_{\omega}$ and $W_{\theta}$ represent standard and independent Wiener processes,

$$
\begin{aligned}
f_{\omega}= & \frac{\omega\left(\alpha_{11}-\alpha_{22}\right)}{2}+\frac{5 \omega\left(\sigma_{11}^{2}+\sigma_{21}^{2}\right)}{8}-\frac{\omega \sigma_{11} \sigma_{21}}{4} \\
& +\frac{\sigma_{11}^{2}+\sigma_{12}^{2}+\sigma_{21}^{2}+\sigma_{22}^{2}}{2 \omega}, \\
f_{\theta}= & -\frac{\alpha_{12}}{2}, \\
\beta_{11}^{2}= & \frac{3 \omega^{2}\left(\sigma_{11}^{2}+\sigma_{21}^{2}\right)}{8}+\frac{\sigma_{11}^{2}+\sigma_{12}^{2}+\sigma_{21}^{2}+\sigma_{22}^{2}}{2}+\frac{\omega^{2} \sigma_{11} \sigma_{21}}{4}, \\
\beta_{12}= & 0, \\
\beta_{21}= & 0, \\
\beta_{22}^{2}= & \frac{\left(\sigma_{21}-\sigma_{11}\right)^{2}}{8}+\frac{\sigma_{11}^{2}+\sigma_{12}^{2}+\sigma_{21}^{2}+\sigma_{22}^{2}}{2 \omega^{2}} .
\end{aligned}
$$

Furthermore, some parameter transformations are provided as follows:

$$
\begin{aligned}
& \epsilon_{1}=\frac{\alpha_{11}+\alpha_{21}}{2}, \\
& \epsilon_{2}=5\left(\sigma_{11}^{2}+\sigma_{21}^{2}\right)-2 \sigma_{11} \sigma_{21}, \\
& \epsilon_{3}=\frac{\sigma_{11}^{2} x_{1}^{* 2}+\sigma_{21}^{2} x_{2}^{* 2}+\sigma_{12}^{2}+\sigma_{22}^{2}}{2}, \\
& \epsilon_{4}=3\left(\sigma_{11}^{2}+\sigma_{21}^{2}\right)+2 \sigma_{11} \sigma_{21}, \\
& \epsilon_{5}=\frac{\alpha_{11}+\alpha_{21}}{4}, \\
& \epsilon_{6}=\left(\sigma_{11}-\sigma_{21}\right)^{2} .
\end{aligned}
$$

According to $\beta_{12}=\beta_{21}=0$ and (31), system (29) can be rewritten as follows:

$$
\begin{aligned}
& \mathrm{d} \omega=\frac{\omega^{2}\left(8 \epsilon_{1}+\epsilon_{2}\right)+8 \epsilon_{3}}{8 \omega} \mathrm{d} t+\sqrt{\frac{8 \epsilon_{3}+\epsilon_{4} \omega^{2}}{8}} \mathrm{~d} W_{\omega}+\sqrt{\epsilon_{5} \omega} \mathrm{d} W_{\theta}, \\
& \mathrm{d} \theta=\sqrt{\epsilon_{5} \omega} \mathrm{d} W_{\omega}+\frac{\sqrt{16 \epsilon_{3}+\epsilon_{6} \omega^{2}}}{4 \omega} \mathrm{d} W_{\theta} .
\end{aligned}
$$

It follows from the above analysis and $\beta_{12}=\beta_{21}=0$ that averaging amplitude $\omega(t)$ refers to the one-dimensional Markov diffusion process, which derives that

$$
\mathrm{d} \omega=\frac{\omega^{2}\left(8 \epsilon_{1}+\epsilon_{2}\right)+8 \epsilon_{3}}{8 \omega} \mathrm{d} t+\sqrt{\frac{8 \epsilon_{3}+\epsilon_{4} \omega^{2}}{8}} \mathrm{~d} W_{\omega} .
$$

Theorem 3. When $\tau_{1}=\tau_{2}=0$ and $\gamma_{i}(u)=0(i=1,2)$, stochastic stability of system (26) is discussed due to the variation of stability of averaging amplitude $\omega(t)$ in terms of probability.

(i) $\omega=0$ is unstable, and system (26) is unstable around $\left(x_{1}^{*}, x_{2}^{*}\right)$ in terms of probability.

(ii) If $2\left(8 \epsilon_{1}+\epsilon_{2}\right)-\epsilon_{4}>0$, then $\omega=+\infty$ is attractively natural and system (26) is unstable around $\left(x_{1}^{*}, x_{2}^{*}\right)$ in terms of probability; system (26) does not undergo stochastic Hopf bifurcation around $\left(x_{1}^{*}, x_{2}^{*}\right)$.

(iii) If $2\left(8 \epsilon_{1}+\epsilon_{2}\right)-\epsilon_{4}=0$, then $\omega=+\infty$ is strictly natural.

(iv) If $2\left(8 \epsilon_{1}+\epsilon_{2}\right)-\epsilon_{4}<0$, then $\omega=+\infty$ is exclusively natural and system (26) is unstable around $\left(x_{1}^{*}, x_{2}^{*}\right)$ in terms of probability.

Proof 5. It follows from the formulation of $\epsilon_{3}$ that $\epsilon_{3}>0$ and $\beta_{11} \neq 0$, which derives that $\omega=0$ is a regular boundary of system (29) (reachable) and $\omega=0$ is unstable. Hence, system (26) is unstable around $\left(x_{1}^{*}, x_{2}^{*}\right)$, which is not relevant to the local stability of the deterministic system around $\left(x_{1}^{*}, x_{2}^{*}\right)$. On the other hand, if $\omega=+\infty$, then $f_{\omega}=\infty$, which derives that $\omega=+\infty$ is the second singular boundary of system (29). Based on the singular boundary theory $[36,37]$, it is easy to show that diffusion exponent $\rho_{1}$, drifting exponent $\rho_{2}$, and characteristic value $\rho_{3}$ of boundary $\omega=+\infty$ are computed as follows:

$$
\begin{aligned}
\rho_{1} & =2, \\
\rho_{2} & =1, \\
\rho_{3} & =-\lim _{\omega \rightarrow+\infty} \frac{2 f_{\omega} \omega^{\rho_{1}-\rho_{2}}}{\beta_{11}^{2}} \\
& =-\lim _{\omega \rightarrow+\infty} \frac{2\left[8 \epsilon_{3}+\omega^{2}\left(8 \epsilon_{1}+\epsilon_{2}\right)\right]}{8 \epsilon_{3}+\omega^{2} \epsilon_{4}} \\
& =-\frac{2\left(8 \epsilon_{1}+\epsilon_{2}\right)}{\epsilon_{4}} .
\end{aligned}
$$

If $2\left(8 \epsilon_{1}+\epsilon_{2}\right)-\epsilon_{4}>0$, then $\omega=+\infty$ is attractively natural and system (26) is unstable around $\left(x_{1}^{*}, x_{2}^{*}\right)$; system (26) does not undergo stochastic Hopf bifurcation around $\left(x_{1}^{*}, x_{2}^{*}\right)$.

If $2\left(8 \epsilon_{1}+\epsilon_{2}\right)-\epsilon_{4}=0$, then $\omega=+\infty$ is strictly natural, which is just the line of stochastic stability demarcation.

If $2\left(8 \epsilon_{1}+\epsilon_{2}\right)-\epsilon_{4}<0$, then $\omega=+\infty$ is exclusively natural and system $(26)$ is unstable around $\left(x_{1}^{*}, x_{2}^{*}\right)$ in terms of probability.

In the following part, existence of stochastic Hopf bifurcation will be investigated as $\omega=+\infty$ is exclusively natural. By using Itô stochastic equation of amplitude of $\omega(t)$, Fokker-Planck equation can be obtained as follows: 


$$
\frac{\partial p}{\partial t}=-\frac{\partial}{\partial \omega} \frac{\left[\left(8 \epsilon_{1}+\epsilon_{2}\right) \omega^{2}+8 \epsilon_{3}\right] p}{8 \omega}+\frac{\partial^{2}}{\partial \omega^{2}} \frac{\left(8 \epsilon_{3}+\omega^{2} \epsilon_{4}\right) p}{16},
$$

with the initial value condition

$$
p\left(\omega, t \mid \omega_{0}, t_{0}\right) \longrightarrow \delta\left(\omega-\omega_{0}\right), \quad t \longrightarrow t_{0},
$$

where $p\left(\omega, t \mid \omega_{0}, t_{0}\right)$ denotes the transition probability density of the diffusion process $\omega(t)$.

By virtue of (35), it follows from simple computations that invariant measure of $\omega(t)$ is the steady-state probability density $p_{s}(\omega)$, which is as follows:

$$
p_{s}(\omega)=\frac{8 \epsilon_{4} \sqrt{2 \pi \epsilon_{3} \epsilon_{4}} \Gamma(2-\kappa)}{\pi\left(8 \epsilon_{3}\right)^{\kappa} \Gamma((1 / 2)-\kappa)} \omega^{2}\left(\epsilon_{4} \omega^{2}+8 \epsilon_{3}\right)^{\kappa-2},
$$

where $\kappa=\left(8 \epsilon_{1}+\epsilon_{2}\right) / \epsilon_{4}$ and $\Gamma(s)=\int_{0}^{+\infty} t^{s-1} e^{-t} \mathrm{~d} t$.

Theorem 4. When $\tau_{1}=\tau_{2}=0$ and $\gamma_{i}(u)=0(i=1,2)$, if $2\left(8 \epsilon_{1}+\epsilon_{2}\right)-\epsilon_{4}<0$ and $8 \epsilon_{1}+\epsilon_{2}-\epsilon_{4}<0$, then there exists $a$ maximum value $\omega=\omega^{*}$ for $p_{s}(\omega)$. Occurrence positions and probabilities of stochastic Hopf bifurcation vary due to values of $\epsilon_{i}, i=1,2,3,4$.
Proof 6. By using Namachivaya's theory [36, 37], it is easy to show that characteristic information of a steady state can be revealed based on the invariant measure. When intension of Gaussian white noises tends to zero, the dynamical behavior of the corresponding deterministic system can be approximated by extreme values of $p_{s}(\omega)$. Based on Oseledec ergodic's theory $[36,37], p_{s}(\omega)$ is considered to be the time measurement in the neighbourhood of $\omega(t)$, which derives that $\epsilon_{1}<0, \epsilon_{2}>0, \epsilon_{3}>0$, and $\epsilon_{4}>0$.

If there exists a maximum value of $p_{s}(\omega)$ around $\zeta^{*}>0$, then it is easy to show that $\zeta^{*}$ is asymptotically stable in terms of probability. On the other hand, if there exists a minimum value of $p_{s}(\omega)$ (zero), then the opposite case also holds. It is assumed that there exists a maximum value as $\omega=\tilde{\omega}$ for $p_{s}(\omega)$; further computations show that

$$
\begin{aligned}
& \left.\frac{\mathrm{d} p_{s}(\omega)}{\mathrm{d} \omega}\right|_{\omega=\tilde{\omega}}=0, \\
& \left.\frac{\mathrm{d}^{2} p_{s}(\omega)}{\mathrm{d} \omega^{2}}\right|_{\omega=\tilde{\omega}}<0,
\end{aligned}
$$

and solutions of the above equation are computed as follows: $\omega=0$ or $\omega=\tilde{\omega}=\sqrt{\left(-8 \epsilon_{3} / 8 \epsilon_{1}+\epsilon_{2}-\epsilon_{4}\right)}$ as $8 \epsilon_{1}+\epsilon_{2}-\epsilon_{4}<0$. Furthermore, it can be computed that

$$
\begin{aligned}
& \left.\frac{\mathrm{d}^{2} p_{s}(\omega)}{\mathrm{d} \omega^{2}}\right|_{\omega=0}=2^{7+3\left(8 \epsilon_{1}+\epsilon_{2}-4 \epsilon_{4}\right) \epsilon_{4}^{-1}} \epsilon_{3}^{2+\left(8 \epsilon_{1}+\epsilon_{2}-4 \epsilon_{4}\right) \epsilon_{4}^{-1}}>0, \\
& \left.\frac{\mathrm{d}^{2} p_{s}(\omega)}{\mathrm{d} \omega^{2}}\right|_{\omega=\tilde{\omega}}=-\frac{\left(8 \epsilon_{1}+\epsilon_{2}-\epsilon_{4}\right)^{3}\left(8 \epsilon_{3}-\left(8 \epsilon_{3} \epsilon_{4} /\left(8 \epsilon_{1}+\epsilon_{2}-\epsilon_{4}\right)\right)\right)^{\left(8 \epsilon_{1}+\epsilon_{2}\right) / \epsilon_{4}}}{16 \epsilon_{3}^{2}\left(8 \epsilon_{1}+\epsilon_{2}-2 \epsilon_{4}\right)^{3}}<0 .
\end{aligned}
$$

It is easy to show that $p_{s}(\omega)=0$ when $\omega=0$. Based on the singular boundary theory $[36,37]$, it shows that system (33) is unstable around $\omega=0$ in terms of probability. Hence, when $\tau_{1}=\tau_{2}=0$ and $\gamma_{i}(u)=0(i=1,2)$, system (26) undergoes stochastic Hopf bifurcation around $\omega=\tilde{\omega}$, which derives that

$$
x_{1}^{2}(t)+x_{2}^{2}(t)=-\frac{8 \epsilon_{3}}{8 \epsilon_{1}+\epsilon_{2}-\epsilon_{4}}, \quad \text { when } \omega=\tilde{\omega} .
$$

Remark 3. From practical and physical perspective of viewpoints, if the proposed hybrid delayed stochastic bioeconomic system is unstable around the interior equilibrium in terms of probability, then it practically implies that the population density of the prey and predator population will not eventually reach an ecological balance state with a high probability, which is not beneficial to sustainable survival of each population under a commercially harvested ecological environment. If stochastic Hopf bifurcation does not occur and boundary is attractively natural, then it practically interprets that either the prey or the predator population density will sharply increase with a high probability within a short duration, which may result in the corresponding shortage of survival space and resource and beyond environment carrying capacity. The ecological balance state will be destroyed in a high probability.

3.2. Case II: System (3) with Double Time Delays and Lévy Jumps. It should be noted that $\left(x_{1}^{*}, x_{2}^{*}\right)$ is not the equilibrium of the stochastic system (26); we will show that solution of system (26) is going around $\left(x_{1}^{*}, x_{2}^{*}\right)$ under certain conditions.

Theorem 5. For $\left(\tau_{1}, \tau_{2}, v\right) \in H_{1} \cap H_{2}$, when $\int_{\mathbb{Y}}\left[\gamma_{i}(u)-\ln \right.$ $\left.\left(1+\gamma_{i}(u)\right)\right] \lambda \mathrm{d} u \leq \tilde{\gamma}_{i}(i=1,2)$ and $\tilde{\gamma}_{i}$ are positive constants and $\sigma_{j k}>0(j, k=1,2)$ is sufficiently small, then

$$
\begin{aligned}
& \limsup _{t \rightarrow \infty} \frac{1}{t} \mathbb{E} \int_{0}^{t}\left[x_{1}(s)-x_{1}^{*}\right]^{2} \mathrm{~d} s \leq B_{1}, \\
& \limsup _{t \rightarrow \infty} \frac{1}{t} \mathbb{E} \int_{0}^{t}\left[x_{2}(s)-x_{2}^{*}\right]^{2} \mathrm{~d} s \leq B_{2},
\end{aligned}
$$



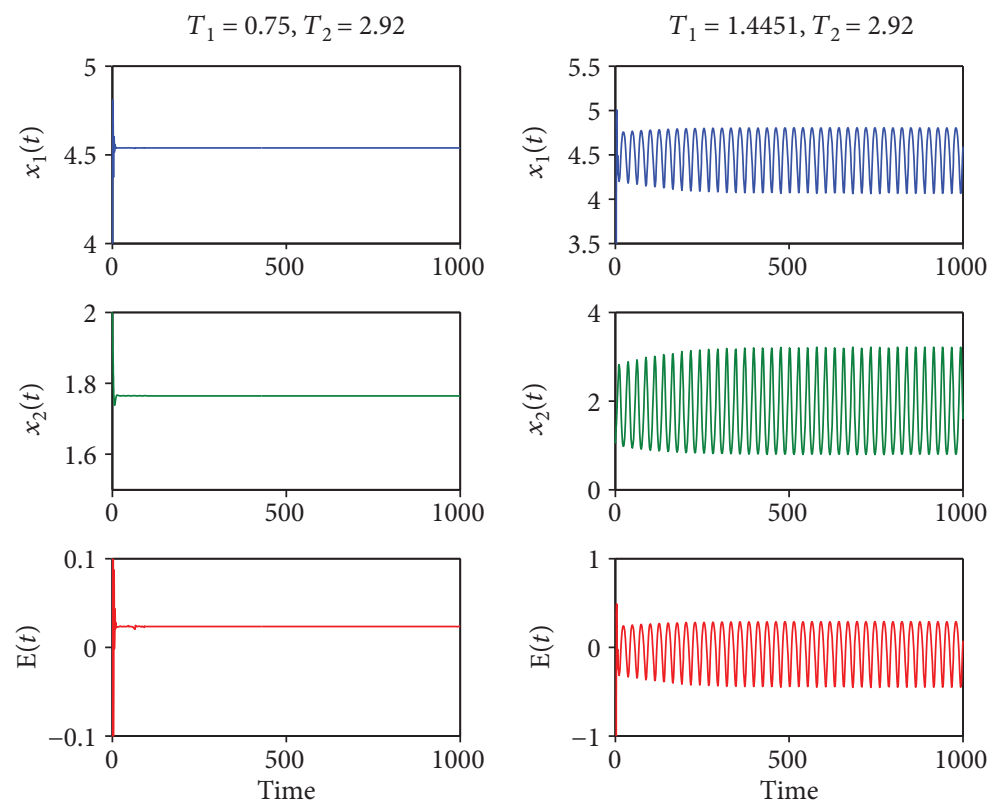

(a)

(b)

Figure 1: Parameter values are given as follows: $r_{1}=2, b_{1}=0.4, a_{1}=0.5, k=6, q=0.1, m_{1}=1, m_{2}=2, r_{2}=0.3, a_{2}=2, w=5, c=1$, and $v=0.5$. When $\sigma_{j k}=0(j, k=1,2)$ and $\gamma_{i}(u)=0(i=1,2)$, dynamical responses of system $(21)$ are plotted, where (a) $\tau_{1}=0.75, \tau_{2}=2.92$, and system $(21)$ is locally asymptotically stable around $(4.5384,1.7647,0.0231)$ and (b) $\tau_{1}=1.4451, \tau_{2}=2.92$, and system (21) is unstable stable around $(4.5384,1.7647,0.0231)$.

where $B_{1}$ and $B_{2}$ are defined as follows:

$$
\begin{aligned}
B_{1}= & \frac{a_{1} m_{1} k P_{1}\left[x_{1}^{*} P_{1}+x_{2}^{*}\left(1+P_{1}\right)\right]+\left(k+x_{1}^{*}\right)\left[q k\left(P+x_{1}^{*}\right)+a_{1} m_{1} P_{2} x_{1}^{*}\right]}{k m_{1} b_{1}\left(k+x_{1}^{*}\right)}+\frac{x_{1}^{*}\left(Q_{1} x_{1}^{*}+P_{1}^{2}\right)}{Q_{1}} \\
& +\frac{2 q v Q_{1}^{2}\left(P_{1}^{2}+x_{1}^{* 2}\right)+m_{2} x_{1}^{*}\left(w Q_{1}-c\right)\left[x_{1}^{*}\left(1+x_{1}^{*}\right) \sigma_{11}^{2}+\left(2+Q_{1}^{2}\right) \sigma_{12}^{2}+2 Q_{1}^{2}\left(1+x_{1}^{*}\right) \tilde{\gamma}_{1}\right]}{2 b_{1} m_{2} x_{1}^{*} Q_{1}^{2}\left(w Q_{1}-c\right)}, \\
B_{2}= & \frac{P_{2}\left(k+P_{1}\right)\left\{\left[\left(a_{2} P_{2}+x_{2}^{*}\right)\left(k+P_{1}\right)+P_{1} P_{2}+x_{1}^{*} x_{2}^{*}\right]+a_{2} P_{2}\left[k P_{2}\left(1+x_{2}^{*}\right)+x_{2}^{*}\left(P_{1}+P_{2}\right)\right]\right\}}{a_{2}\left(k+Q_{1}\right)\left[k\left(1+Q_{2}\right)+Q_{1}\right]}+\frac{x_{2}^{*} \sigma_{21}^{2}\left(\sigma_{22}^{2}+2 x_{2}^{*}\right)+\sigma_{22}^{2}+4 \tilde{\gamma}_{2}}{2 a_{2}\left[k\left(1+Q_{2}\right)+Q_{1}\right]} .
\end{aligned}
$$

Proof 7. The proof of Theorem 5 can be found in Appendix C of this paper.

\section{Numerical Simulation}

Numerical simulations are carried out to show combined dynamic effects of double time delays and Lévy jumps on population dynamics. Parameters are partially taken from numerical simulations in [18], $r_{1}=2, b_{1}=0.4, a_{1}=$ $0.5, k=6, q=0.1, m_{1}=1, m_{2}=2, r_{2}=0.3, a_{2}=2, w=5$, and $c=1$ with appropriate units.
4.1. Numerical Simulation for Deterministic System. By utilizing the above parameter values, it follows from (21) that there exists an interior equilibrium when $0<v<2.38$. In order to facilitate the following analysis, $v=0.5$ is arbitrarily selected within $(0,2.38)$ which is enough to merit the corresponding analysis in this paper, and an interior equilibrium $M^{*}(4.5384,1.7647,0.0231)$ can be obtained as $v=0.5$. Based on Theorem 1 and (A.10), if $0<\tau_{1}<\tau_{1}^{*}=1.4451$ and $0<\tau_{2}<\tau_{2}^{*}=6.4223$, then it is easy to show that interior equilibrium $M^{*}(4.5384,1.7647,0.0231)$ of system (22) is asymptotically stable. Based on the above analysis, some numerical simulations are supported. System 

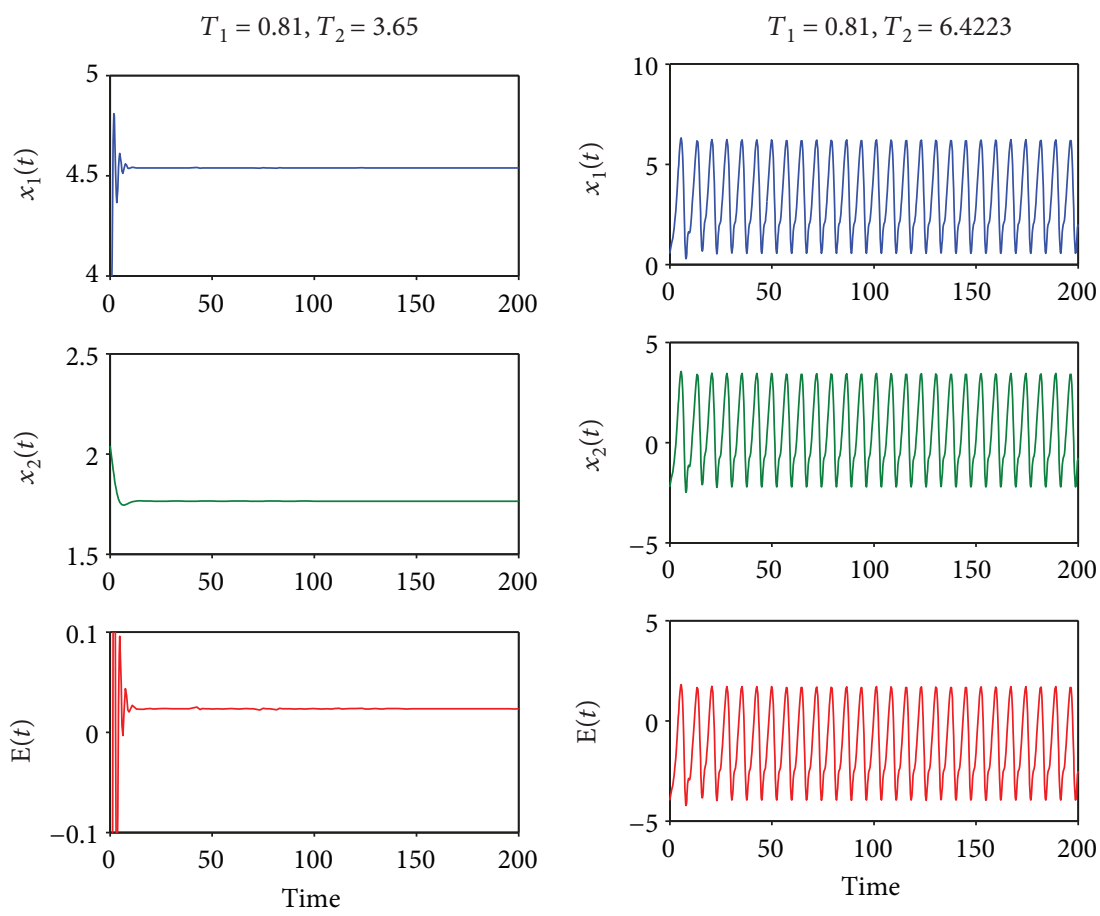

(a)

(b)

Figure 2: Parameter values are given as follows: $r_{1}=2, b_{1}=0.4, a_{1}=0.5, k=6, q=0.1, m_{1}=1, m_{2}=2, r_{2}=0.3, a_{2}=2, w=5, c=1$, and $v=0.5$. When $\sigma_{j k}=0(j, k=1,2)$ and $\gamma_{i}(u)=0(i=1,2)$, dynamical responses of system (21) are plotted, where $(\mathrm{a}) \tau_{1}=0.81, \tau_{2}=3.65$, and system (21) is locally asymptotically stable around $(4.5384,1.7647,0.0231)$ and (b) $\tau_{1}=0.81, \tau_{2}=6.4223$, and system (21) is unstable stable around $(4.5384,1.7647,0.0231)$.

TABle 1: Probabilities and occurrence positions of stochastic Hopf bifurcation of system (26) with $\sigma_{11}=0.1$ and $\sigma_{21}=0.5$ and different values of $\sigma_{12}$ and $\sigma_{22}$.

\begin{tabular}{lcccc}
\hline & $\sigma_{12}=\sigma_{22}=0.1$ & $\sigma_{12}=\sigma_{22}=0.2$ & $\sigma_{12}=\sigma_{22}=0.3$ & $\sigma_{12}=\sigma_{22}=0.5$ \\
\hline$\epsilon_{1}$ & -0.8982 & -0.8982 & -0.8982 & -0.8982 \\
$\epsilon_{2}$ & 1.2 & 1.2 & 1.2 & 1.2 \\
$\epsilon_{3}$ & 0.5023 & 0.5323 & 0.5823 & 0.7423 \\
$\epsilon_{4}$ & 0.88 & 0.88 & 0.88 & 0.88 \\
$\tilde{\omega}$ & 0.7650 & 0.7876 & 0.8237 & 0.93 \\
$p_{s}(\tilde{\omega})$ & 0.9705 & 0.9427 & 0.9013 & 0.7983 \\
\hline
\end{tabular}

(22) with $\tau_{1}=0.75<\tau_{1}^{*}$ and $\tau_{2}=2.92$ is locally asymptotically stable around $M^{*}(4.5384,1.7647,0.0231)$, whose dynamical responses are plotted in Figure 1(a). System (22) with $\tau_{1}=\tau_{1}^{*}=1.4451$ and $\tau_{2}=2.92$ is unstable around $M^{*}(4.5384,1.7647,0.0231)$, whose dynamical responses are plotted in Figure 1(b). Furthermore, system (22) with $\tau_{1}=0.81$ and $\tau_{2}=3.65<\tau_{2}^{*}$ is locally asymptotically stable around $M^{*}(4.5384,1.7647,0.0231)$, whose dynamical responses are plotted in Figure 2(a). System (22) with $\tau_{1}=0.81$ and $\tau_{2}=\tau_{2}^{*}=6.4223$ is unstable around $M^{*}(4.5384,1.7647,0.0231)$, whose dynamical responses are plotted in Figure 2(b).
4.2. Numerical Simulation for Stochastic System. In the absence of double time delays and Lévy jumps, by taking $\sigma_{11}=0.1$ and $\sigma_{21}=0.5$ as fixed values, it follows from simple computations that $\epsilon_{1}=-0.8982, \epsilon_{2}=1.2$, and $\epsilon_{4}=0.88$, which derive $2\left(8 \epsilon_{1}+\epsilon_{2}\right)-\epsilon_{4}=-12.8512<0$ and $8 \epsilon_{1}+$ $\epsilon_{2}-\epsilon_{4}=-6.8656<0$. Based on Theorems 3 and 4 of this paper, when $v=0.5$, probabilities and occurrence positions of stochastic Hopf bifurcation of system (27) with $\sigma_{11}=0.1$ and $\sigma_{21}=0.5$ and different values of $\sigma_{12}=\sigma_{22}=$ $0.1,0.2,0.3,0.5$ are computed in Table 1 . Corresponding to the computation results in Table 1, the probability density curve $p_{s}(\omega)$ and occurrence positions of stochastic 


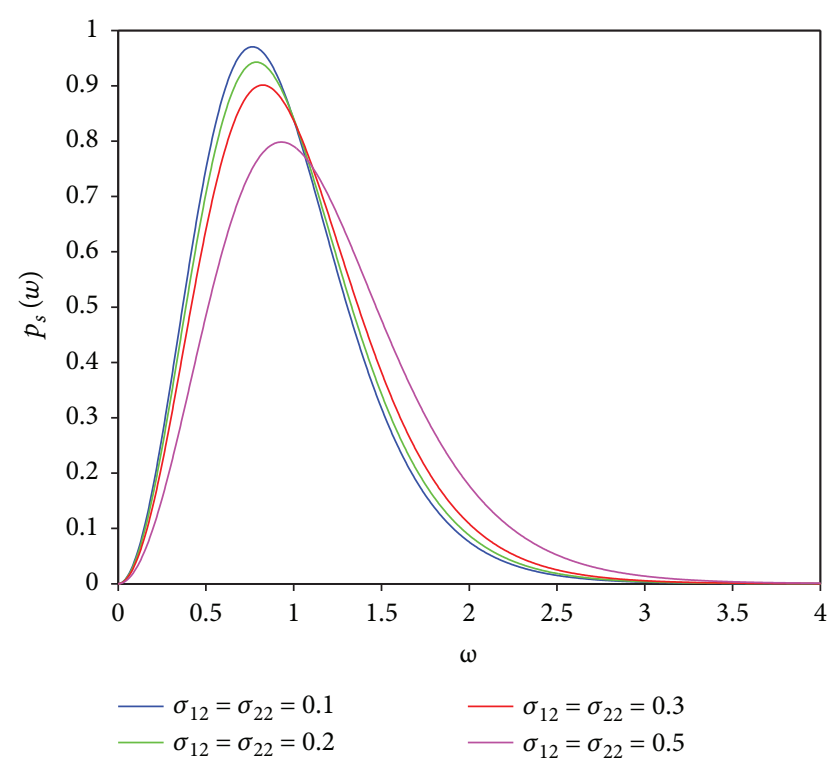

FIGURE 3: Parameter values for numerical simulations are given as follows: $r_{1}=2, b_{1}=0.4, a_{1}=0.5, k=6, q=0.1, m_{1}=1, m_{2}=2$, $r_{2}=0.3, a_{2}=2, w=5, c=1$, and $v=0.5$. When $\gamma_{i}(u)=0(i=1,2)$ and $\tau_{1}=\tau_{2}=0$, the probability densities $p_{s}(\omega)$ and occurrence positions of stochastic Hopf bifurcation of system (27) with $\sigma_{11}=$ 0.1 and $\sigma_{21}=0.5$ and different values of $\sigma_{12}$ and $\sigma_{22}$, where (a) $\sigma_{12}=\sigma_{22}=0.1$, (b) $\sigma_{12}=\sigma_{22}=0.2$, (c) $\sigma_{12}=\sigma_{22}=0.3$, and (d) $\sigma_{12}=\sigma_{22}=0.5$.

Hopf bifurcation of system (27) due to variations of $\omega$ are plotted in Figure 3. According to (40), the corresponding phase portraits of probability densities $p_{s}(\omega)$ with respect to $x_{1}(t)$ and $x_{2}(t)$ are plotted in Figure 4.

In the presence of double time delays and Lévy jumps, if $0<\tau_{1}<\tau_{1}^{*}$ and $0<\tau_{2}<\tau_{2}^{*}$, then it follows from Theorem 5 that $\lim \sup _{t \rightarrow \infty}(1 / t) \mathbb{E} \int_{0}^{t}\left[x_{1}(s)-x_{1}^{*}\right]^{2} \mathrm{~d} s \leq B_{1}$ and $\lim \sup _{t \rightarrow \infty}$ $(1 / t) \mathbb{E} \int_{0}^{t}\left[x_{2}(s)-x_{2}^{*}\right]^{2} \mathrm{~d} s \leq B_{2}$, where $B_{1}$ and $B_{2}$ have been defined in Theorem 5. It follows from the mathematical form of $B_{1}$ and $B_{2}$ that $\sigma_{i j}$ and $\gamma_{i}(i, j=1,2)$ affect asymptotic behaviors of system (3) around interior equilibrium $M^{*}$ as well as fluctuation intensity of population densities of $x_{1}(t)$ and $x_{2}(t)$. By assuming $\tau_{1}=0.8, \tau_{2}=3.5, v=0.5, \sigma_{11}=0.1$, and $\sigma_{21}=0.5$, dynamical responses of system (3) with $\sigma_{12}=$ 0.1 and $\sigma_{22}=0.1$ and different values of $\gamma_{1}$ and $\gamma_{2}$ are obtained based on Euler-Maruyama method and plotted in Figure 5, where $\gamma_{1}=\gamma_{2}=0.1$ and $\gamma_{1}=\gamma_{2}=0.5$. Similarly, by assuming $\tau_{1}=0.8, \tau_{2}=3.5, v=0.5, \sigma_{11}=0.1$, and $\sigma_{21}=0.5$, dynamical responses of system (3) with $\sigma_{12}=0.5$ and $\sigma_{22}=$ 0.5 and different values of $\gamma_{1}$ and $\gamma_{2}$ are obtained based on Euler-Maruyama method and plotted in Figure 6, where $\gamma_{1}=\gamma_{2}=0.1$ and $\gamma_{1}=\gamma_{2}=0.5$.

Based on the numerical simulations in Figures 1 and 2, it follows that local asymptotic stability of system (22) around interior equilibrium switches due to variations of time delays. System (22) shows unstable dynamical responses when time delays cross critical values, which can be computed based on Theorem 1. It follows from the mathematical formulation of $\epsilon_{3}$ and numerical computational results in Table 1 that the value of $\epsilon_{3}$ increases as intensities of external excitations $\sigma_{12}$ and $\sigma_{22}$ values increase. Furthermore, it follows from Figure 3 the occurrence positions where system (27) undergoes stochastic Hopf bifurcation switch higher as the intensities of external excitations $\sigma_{12}$ and $\sigma_{22}$ values increase. On the other hand, the corresponding stochastic Hopf bifurcation will decrease in a higher probability, which is observed in Figure 4 . Based on the numerical simulations in Figures 5 and 6 , it can be concluded that the magnitude of environmental external stochastic excitation $\sigma_{12}$ and $\sigma_{22}$ values and Lévy jump $\gamma_{1}$ and $\gamma_{2}$ values plays significant roles to determine the magnitude of oscillation of population dynamics. It follows from the detailed numerical comparison results between Figures 5 and 6 that oscillation magnitude of dynamical responses increases as Lévy jump $\gamma_{1}$ and $\gamma_{2}$ values increase for the fixed environmental external stochastic excitation $\sigma_{12}$ and $\sigma_{22}$ values. On the other hand, oscillation magnitudes of dynamical responses increase as external stochastic excitation $\sigma_{12}$ and $\sigma_{22}$ values increase for the fixed environmental Lévy jump $\gamma_{1}$ and $\gamma_{2}$ values.

\section{Conclusion}

In this paper, a double delayed hybrid stochastic bioeconomic system with commercial harvesting and Lévy jumps is established, which extends work done in [18] by incorporating double time delays and stochastic fluctuations. The dynamical model proposed in [18] is composed of ordinary differential equations, which are utilized to study the interaction mechanism of the prey-predator system with single time delay. Compared with the system established in [18], an algebraic equation is introduced into system (3), which concentrates on the dynamic effect of the economic interest of commercial harvesting on population dynamics and provides a straightforward way to investigate complex dynamics due to the variation of economic interests. Furthermore, two discrete time delays, which represent the maturation delay of the prey and gestation delay of the predator, are incorporated into system (3). Population growth of the prey and predator population affected by environmental stochastic fluctuations is assumed to be a stochastic process. Gaussian white noises and Lévy jumps are incorporated into system (3) to describe stochastic surrounding environmental factors. For the deterministic system, positivity of solutions and uniform persistence of system are discussed in Lemmas 1 and 2, respectively. Some sufficient conditions associated with double time delays are derived to discuss the asymptotic stability of interior equilibrium in Theorem 1 . For the stochastic system, existence and uniqueness of global positive solution are studied in Theorem 2. By using the invariant measure theory and singular boundary theory of the diffusion process, the existence of stochastic Hopf bifurcation and stochastic stability is investigated in the absence of double time delays and Lévy jumps, which can be found in Theorems 3 and 4, respectively. By 


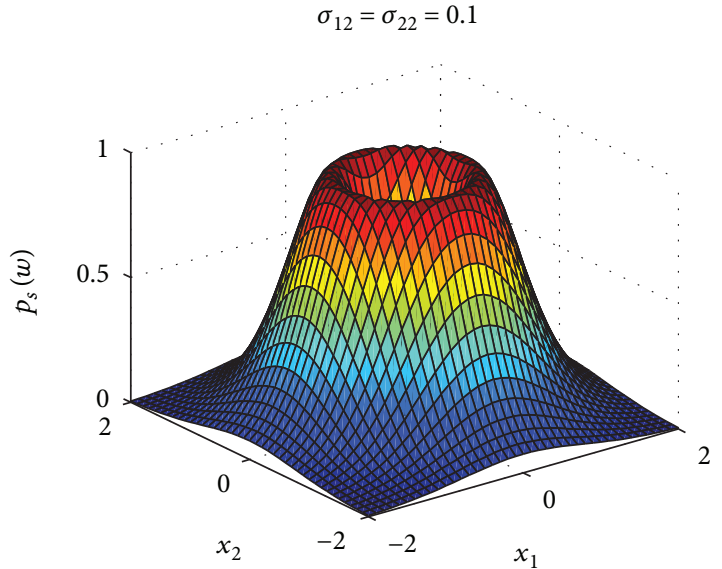

(a)

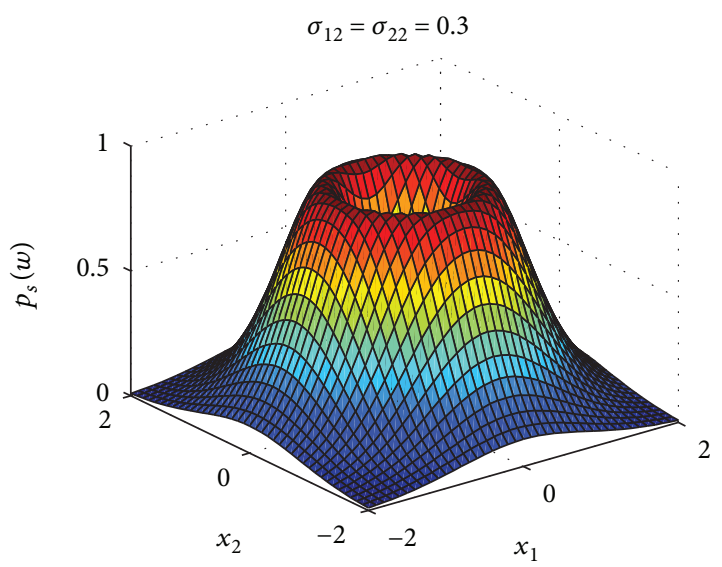

(c)

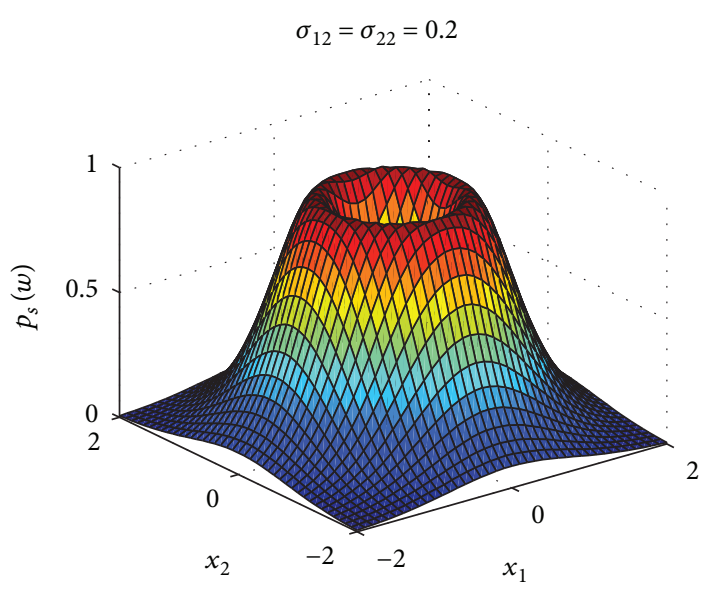

(b)

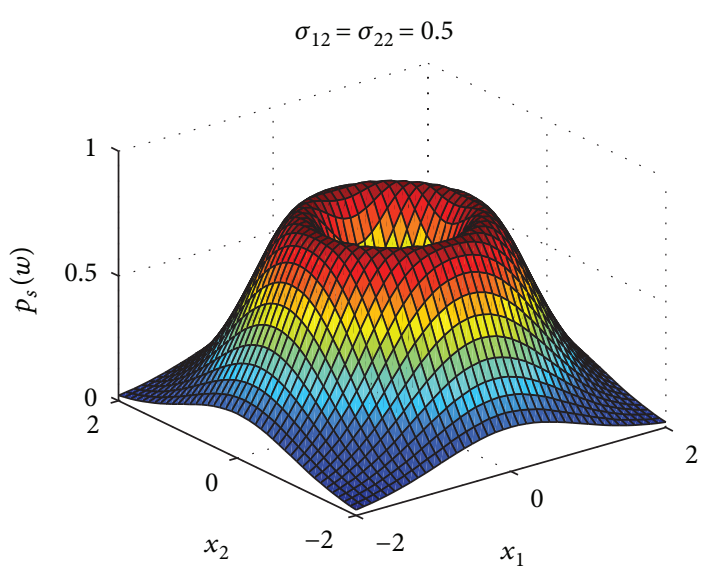

(d)

Figure 4: Parameter values for numerical simulations are given as follows: $r_{1}=2, b_{1}=0.4, a_{1}=0.5, k=6, q=0.1, m_{1}=1, m_{2}=2, r_{2}=0.3$, $a_{2}=2, w=5, c=1$, and $v=0.5$. The phase portraits of probability densities $p_{s}(\omega)$ corresponding to Figure 3 in the $x_{1}-x_{2}-p_{s}(\omega)$ space, where (a) $\sigma_{12}=\sigma_{22}=0.1$, (b) $\sigma_{12}=\sigma_{22}=0.2$, (c) $\sigma_{12}=\sigma_{22}=0.3$, and (d) $\sigma_{12}=\sigma_{22}=0.5$.

constructing appropriate Lyapunov functions, the asymptotic dynamic behavior of the proposed hybrid stochastic system with double time delays and Lévy jumps is discussed in Theorem 5.

From the practical perspective of viewpoints, positivity of the proposed hybrid stochastic double delayed bioeconomic system is relevant to the prey and predator population survival for a long duration under a commercially harvested environment. Generally speaking, the natural resources for the prey and predator population survival are under sever intraspecies competition. Therefore, permanence of the proposed hybrid stochastic double delayed bioeconomic system biologically means that there are certain positively finite upper constraints and positively finite lower constraints for the prey and predator population, which may, to a great extent, avoid population extinction and overpopulation. Furthermore, permanence of the proposed hybrid stochastic double delayed bioeconomic system biologically also means that there exist positively finite upper constraints and positively finite lower constraints for commercial harvesting amount on the prey population. The practical interpretations are introduced as follows: with the purpose of maintaining sustainable development of commercially harvested prey population resources, commercial harvesting should be regulated within certain harvesting range by formulating some constructive policies.

From the practical perspective of viewpoints, if the proposed hybrid stochastic double delayed bioeconomic system is unstable in terms of probability, then it practically interprets that the prey and predator population density will not eventually arrive at an ecological balance level with a high probability. The biological interpretations show that such phenomenon is not advantageous for a sustainable development of interacting populations under a commercial harvest effect. If the boundary is attractively natural and stochastic Hopf bifurcation does not occur, then it practically means that the interacting population densities may dramatically increase with a high probability during short time and 

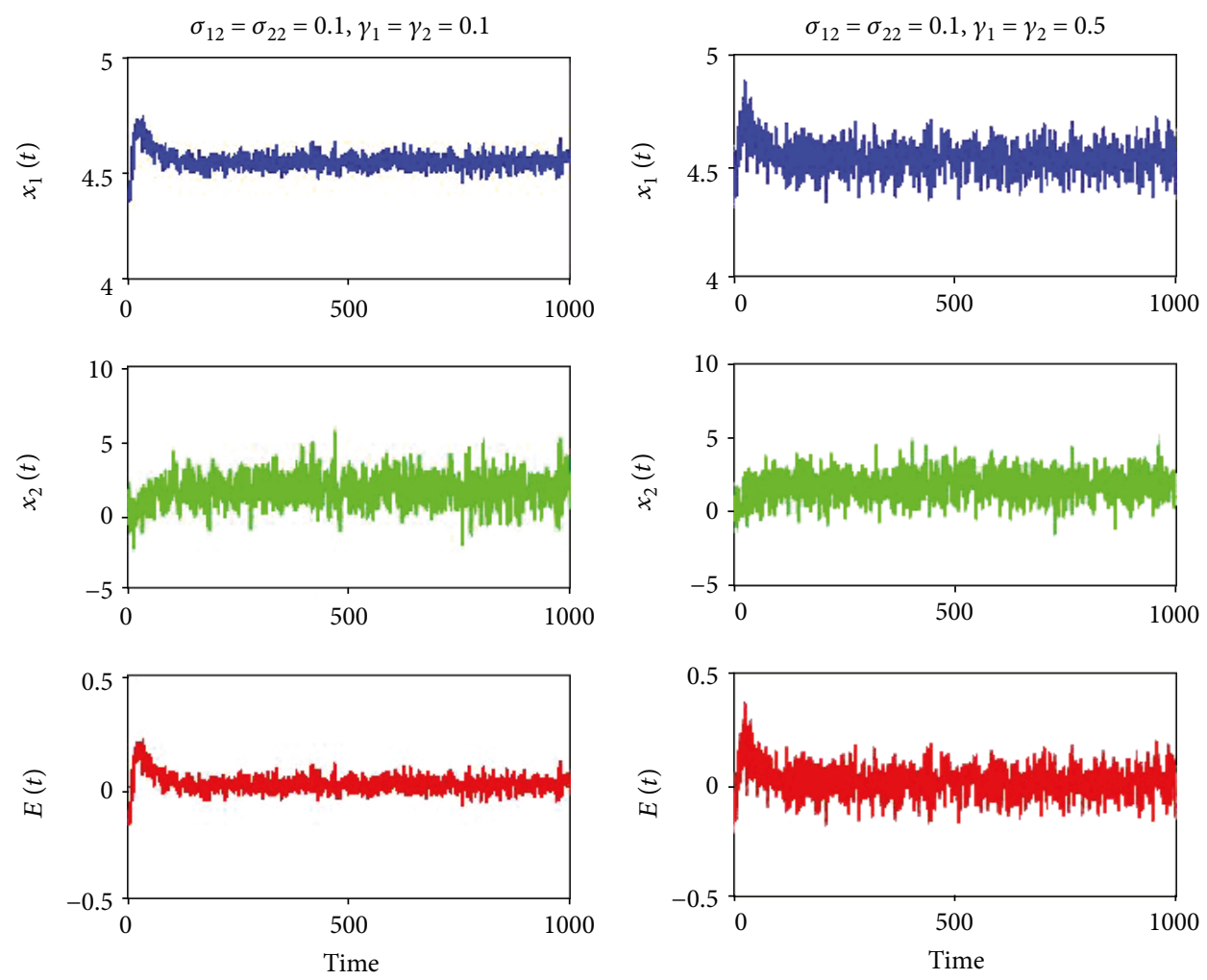

(a)

(b)

Figure 5: Parameter values for numerical simulations are given as follows: $r_{1}=2, b_{1}=0.4, a_{1}=0.5, k=6, q=0.1, m_{1}=1, m_{2}=2, r_{2}=0.3$, $a_{2}=2, w=5, c=1$, and $v=0.5$. Dynamical responses of system (3) with $\sigma_{11}=0.1, \sigma_{21}=0.5, \sigma_{12}=0.1$, and $\sigma_{22}=0.1$ and different values of $\gamma_{1}$ and $\gamma_{2}$, where (a) $\gamma_{1}=\gamma_{2}=0.1$ and (b) $\gamma_{1}=\gamma_{2}=0.5$.

it may reduce the corresponding survival resources and space, which are beyond the environment carrying capacity and destroy the ecological balance state with a high probability.

From the resource management of viewpoints, the numerical simulations reveal that population density may remain an ideal level by controlling double time delays within certain constraints and the critical values of time delays may increase as economic interests of commercial harvesting increase. The sustainable development of commercially harvested population can be indirectly achieved by formulating relevant policy to regulate an economic interest within some appropriate ranges.

Recently, some hybrid bioeconomic systems with time delay and stochastic fluctuations are established in [27-30] to investigate the combined dynamic effects of stochastic fluctuation and commercial harvesting on population dynamics. These proposed systems in [27-30] are also constructed by several differential equations with stochastic fluctuations and an algebraic equation. Compared with the previously bioeconomic systems proposed in $[19-23,31]$ and the references therein, such delayed bioeconomic systems [27-30] can not only discuss coexistence and interaction mechanism of delayed bioeconomic system under stochastic environmental fluctuations but also investigate population dynamics due to variations of the economic interest of commercial harvesting. However, biological characteristics among interacting populations are not considered in [27-29]; time delays such as gestation delay and maturation delay for interacting populations in [27-29] are assumed to be the same discrete value, which contradicts to the reality in the real world. Asymptotical stability of interior equilibrium and dynamic effects of Lévy jumps on population dynamics are not discussed in [27-29]. It is proved that Lévy noise can efficiently depict sudden and severe environmental perturbations arising in the real world [24, 25], while these phenomena can not be described better by Brownian motion. Although the dynamic effects of double time delays have been investigated in [30], the combined dynamic effects of multiple time delays and Lévy jumps on population dynamics have not been investigated in [30]. Dynamic effects of multiple time delays on the hybrid bioeconomic prey-predator system are investigated in $[15,32]$. However, the dynamic effect of Lévy jumps and asymptotical stability of solutions of the stochastic system are not studied in $[15,32]$. To the authors' best knowledge, population dynamics of the hybrid bioeconomic system with double time delays and Lévy jumps 

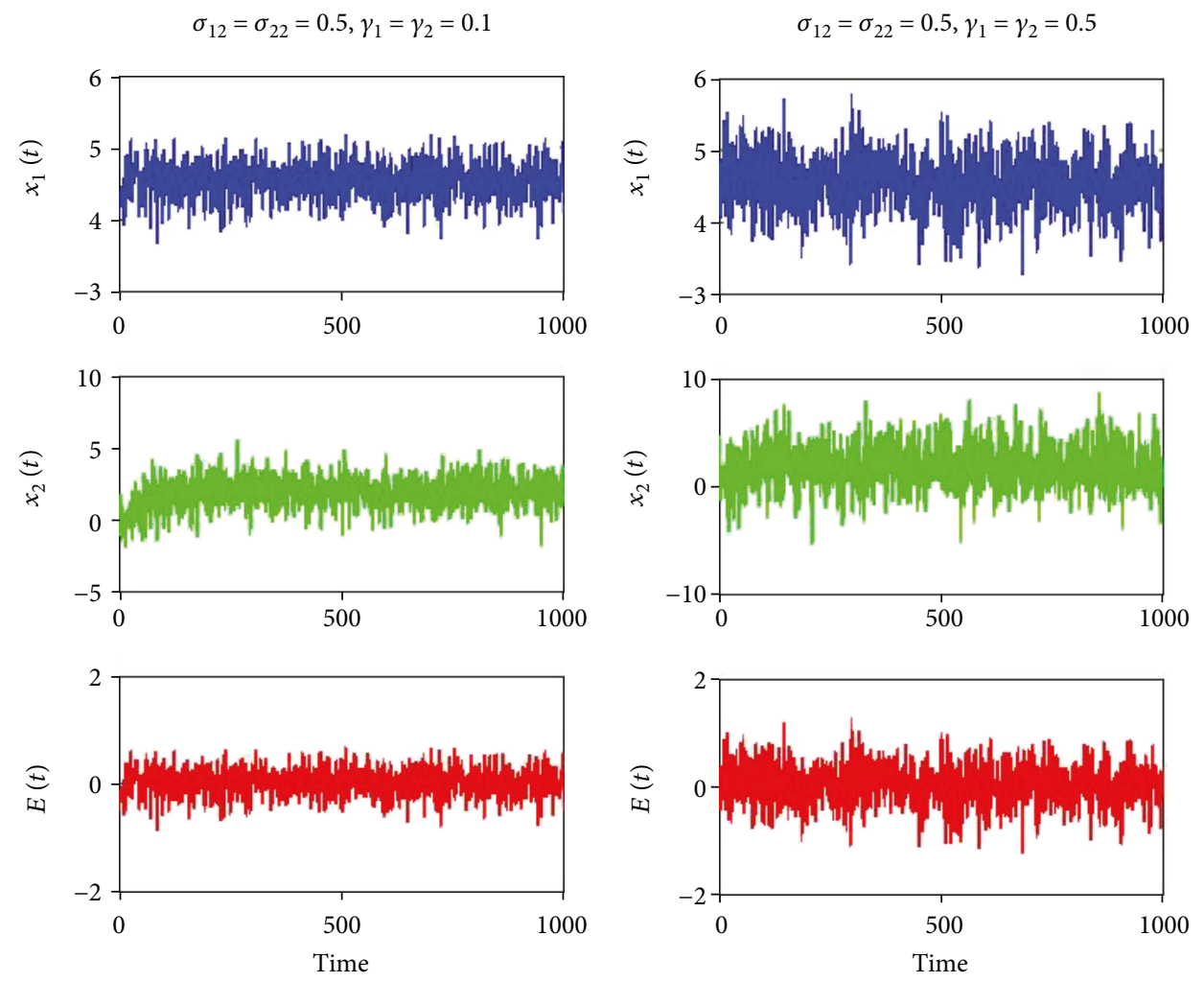

(a)

(b)

FIgURE 6: Parameter values for numerical simulations are given as follows: $r_{1}=2, b_{1}=0.4, a_{1}=0.5, k=6, q=0.1, m_{1}=1, m_{2}=2, r_{2}=0.3$, $a_{2}=2, w=5, c=1$, and $v=0.5$. Dynamical responses of system (3) with $\sigma_{11}=0.1, \sigma_{21}=0.5, \sigma_{12}=0.5$, and $\sigma_{22}=0.5$ and different values of $\gamma_{1}$ and $\gamma_{2}$, where (a) $\gamma_{1}=\gamma_{2}=0.1$ and (b) $\gamma_{1}=\gamma_{2}=0.5$.

have not been investigated. Compared with the related work, we can investigate combined dynamic effects of double time delays and Lévy jumps on population dynamics by analyzing stability analysis and stochastic dynamical behavior of system (3) in this paper; these analytical findings make this paper have some positive and new features.

\section{Appendix}

\section{A. Proof of Theorem 1}

Proof 8 . Let $W_{1}(t)=\left|y_{1}(t)\right|$, by using Lemma 2 and computing the upper right derivative of $W_{1}(t)$ along the solution of system (24), it can be obtained that

$$
\begin{aligned}
D^{+} W_{1}(t) \leq & -\frac{b_{1} x_{1}^{*} P_{1}\left(e^{y_{1}(t)}-1\right)}{Q_{1}}-\frac{a_{1} k x_{2}^{*}\left(e^{y_{2}(t)}-1\right)}{\left(k+Q_{1}\right)\left(k+x_{1}^{*}\right)}+\frac{q v m_{2} x_{1}^{*}\left(w x_{1}^{*}-c\right)\left(e^{y_{1}(t)}-1\right)}{\left[m_{1} v+m_{2} x_{1}^{*}\left(w x_{1}^{*}-c\right)\right]\left[m_{1} v+m_{2} Q_{1}\left(w x_{1}^{*}-c\right)\right]} \\
& +\left(\frac{b x_{1}^{*} P_{1}}{Q_{1}}-\frac{a_{1} x_{1}^{*} x_{2}^{*}}{P_{1}}-\frac{q v x_{1}^{*}}{P_{1}\left[m_{1} v+m_{2} x_{1}^{*}\left(w x_{1}^{*}-c\right)\right]}\right) \int_{t-\tau_{1}}^{t} e^{y_{1}(s)} \frac{\mathrm{d} y_{1}}{\mathrm{~d} s} \mathrm{~d} s \leq-\frac{b_{1} x_{1}^{*} P_{1}\left(e^{y_{1}(t)}-1\right)}{Q_{1}}-\frac{a_{1} k x_{2}^{*}\left(e^{y_{2}(t)}-1\right)}{\left(k+Q_{1}\right)\left(k+x_{1}^{*}\right)} \\
& +\frac{q v m_{2} x_{1}^{*}\left(w x_{1}^{*}-c\right)\left(e^{y_{1}(t)}-1\right)}{\left[m_{1} v+m_{2} x_{1}^{*}\left(w x_{1}^{*}-c\right)\right]\left[m_{1} v+m_{2} Q_{1}\left(w x_{1}^{*}-c\right)\right]}-\left(\frac{b x_{1}^{*} P_{1}}{Q_{1}}-\frac{a_{1} x_{1}^{*} x_{2}^{*}}{P_{1}}-\frac{q v x_{1}^{*}}{P_{1}\left[m_{1} v+m_{2} x_{1}^{*}\left(w x_{1}^{*}-c\right)\right]}\right) \int_{t-\tau_{1}}^{t} b_{1} P_{1}\left(e^{y_{1}\left(s-\tau_{1}\right)}-1\right) \mathrm{d} s \\
& -\left(\frac{b x_{1}^{*} P_{1}}{Q_{1}}-\frac{a_{1} x_{1}^{*} x_{2}^{*}}{P_{1}}-\frac{q v x_{1}^{*}}{P_{1}\left[m_{1} v+m_{2} x_{1}^{*}\left(w x_{1}^{*}-c\right)\right]}\right) \int_{t-\tau_{1}}^{t} \frac{a_{1} x_{2}^{*} k P_{1}\left(e^{y_{2}(s)}-1\right)}{x_{1}^{*}\left(k+x_{1}^{*}\right)\left(k+Q_{1}\right)} \mathrm{d} s+\left(\frac{b x_{1}^{*} P_{1}}{Q_{1}}-\frac{a_{1} x_{1}^{*} x_{2}^{*}}{P_{1}}-\frac{q v x_{1}^{*}}{P_{1}\left[m_{1} v+m_{2} x_{1}^{*}\left(w x_{1}^{*}-c\right)\right]}\right) \\
& \times \int_{t-\tau_{1}}^{t} \frac{q v m_{2} P_{1}\left(w P_{1}(t)-c\right)\left(e^{y_{2}(s)}-1\right)}{\left[m_{1} v+m_{2} x_{1}^{*}\left(w x_{1}^{*}-c\right)\right]\left[m_{1} v+m_{2} Q_{1}\left(w x_{1}^{*}-c\right)\right]} \mathrm{d} s,
\end{aligned}
$$


where $P_{i}, Q_{i}(i=1,2)$ have been defined in Lemma 2 .

Furthermore, let

$$
\begin{aligned}
W_{2}(t)= & W_{1}(t)-P_{1} \tilde{W}_{1} \int_{t-\tau_{1}}^{t} \int_{z}^{t} b_{1}\left|e^{y_{1}\left(s-\tau_{1}\right)}-1\right| \\
& +\frac{a_{1} x_{2}^{*} k\left|e^{y_{2}(s)}-1\right|}{x_{1}^{*}\left(k+x_{1}^{*}\right)\left(k+Q_{1}\right)} \mathrm{d} s \mathrm{~d} z \\
& +\int_{t-\tau_{1}}^{t} \int_{z}^{t} \frac{q v m_{2} P_{1} \tilde{W}_{1}\left(w P_{1}-c\right)\left|e^{y_{1}(s)}-1\right|}{\left[m_{1} v+m_{2} x_{1}^{*}\left(w x_{1}^{*}-c\right)\right]\left[m_{1} v+m_{2} Q_{1}\left(w x_{1}^{*}-c\right)\right]} \mathrm{d} s \mathrm{~d} z
\end{aligned}
$$

where $\quad \tilde{W}_{1}=\left(b x_{1}^{*} P_{1} / Q_{1}\right)-\left(a_{1} x_{1}^{*} x_{2}^{*} / P_{1}\right)-\left(q v x_{1}^{*} /\left(P_{1}\left[m_{1} v+\right.\right.\right.$ $\left.\left.\left.m_{2} x_{1}^{*}\left(w x_{1}^{*}-c\right)\right]\right)\right)$.

By virtue of (A.1) and computing the upper right derivative of $W_{2}(t)$ along the solution of system (24), it can be obtained that

$$
\begin{aligned}
D^{+} W_{2}(t) \leq & D^{+} W_{1}(t)-b_{1} P_{1} \tilde{W}_{1} \tau_{1}\left|e^{y_{1}\left(t-\tau_{1}\right)}-1\right| \\
& -\frac{a_{1} x_{2}^{*} k P_{1} \tilde{W}_{1} \tau_{1}\left|e^{y_{2}(t)}-1\right|}{x_{1}^{*}\left(k+x_{1}^{*}\right)\left(k+Q_{1}\right)} \\
& +\frac{q v m_{2} P_{1} \tilde{W}_{1} \tau_{1}\left(w P_{1}-c\right)\left|e^{y_{1}(t)}-1\right|}{\left[m_{1} v+m_{2} x_{1}^{*}\left(w x_{1}^{*}-c\right)\right]\left[m_{1} v+m_{2} Q_{1}\left(w x_{1}^{*}-c\right)\right]} \\
& +\tilde{W}_{1}\left[\int_{t-\tau_{1}}^{t} b_{1} P_{1}\left(1-e^{y_{1}\left(s-\tau_{1}\right)}\right) \mathrm{d} s\right. \\
& \left.\quad+\int_{t-\tau_{1}}^{t} \frac{a_{1} x_{2}^{*} k P_{1}\left(1-e^{y_{2}(s)}\right)}{x_{1}^{*}\left(k+x_{1}^{*}\right)\left(k+Q_{1}\right)} \mathrm{d} s\right] \\
& -\tilde{W}_{1} \int_{t-\tau_{1}}^{t} \frac{q v m_{2} P_{1}\left(w P_{1}-c\right)\left(e^{y_{2}(s)}-1\right)}{\left[m_{1} v+m_{2} x_{1}^{*}\left(w x_{1}^{*}-c\right)\right]\left[m_{1} v+m_{2} Q_{1}\left(w x_{1}^{*}-c\right)\right]} \mathrm{d} s .
\end{aligned}
$$

Let $W_{3}(t)=\left|y_{2}(t)\right|$, by using Lemma 2 and computing the upper right derivative of $W_{3}(t)$ along the solution of system (24), it can be obtained that

$$
\begin{aligned}
D^{+} W_{3}(t) \leq & \frac{a_{2} x_{1}^{*} x_{2}^{*}\left(e^{y_{1}(t)}-1\right)}{\left(k+x_{1}^{*}\right)\left(k+Q_{1}\right)}-\frac{a_{2} x_{2}^{*}\left(e^{y_{2}(t)}-1\right)}{k+P_{1}}-\frac{a_{2} x_{1}^{*} x_{2}^{*}}{\left(k+x_{1}^{*}\right)\left(k+P_{1}\right)} \int_{t-\tau_{2}}^{t} e^{y_{1}(s)} \frac{\mathrm{d} y_{1}}{\mathrm{~d} s} \mathrm{~d} s+\frac{a_{2} x_{2}^{*}}{k+Q_{1}} \int_{t-\tau_{2}}^{t} e^{y_{2}(s)} \frac{\mathrm{d} y_{2}}{\mathrm{~d} s} \mathrm{~d} s \\
\leq & \frac{a_{2} x_{1}^{*} x_{2}^{*}\left(e^{y_{1}(t)}-1\right)}{\left(k+x_{1}^{*}\right)\left(k+Q_{1}\right)}-\frac{a_{2} x_{2}^{*}\left(e^{y_{2}(t)}-1\right)}{k+P_{1}}+\frac{a_{2} x_{1}^{*} x_{2}^{*}}{\left(k+x_{1}^{*}\right)\left(k+P_{1}\right)} \int_{t-\tau_{2}}^{t} b_{1} P_{1}\left(e^{y_{1}\left(s-\tau_{1}\right)}-1\right) \mathrm{d} s \\
& +\frac{a_{2} x_{1}^{*} x_{2}^{*}}{\left(k+x_{1}^{*}\right)\left(k+P_{1}\right)} \int_{t-\tau_{2}}^{t} \frac{a_{1} x_{2}^{*} k P_{1}\left(e^{y_{2}(s)}-1\right)}{x_{1}^{*}\left(k+x_{1}^{*}\right)\left(k+Q_{1}\right)} \mathrm{d} s-\frac{a_{2} x_{1}^{*} x_{2}^{*}}{\left(k+x_{1}^{*}\right)\left(k+P_{1}\right)} \int_{t-\tau_{2}}^{t} \frac{q v m_{2} P_{1}\left(w P_{1}(t)-c\right)\left(e^{y_{2}(s)}-1\right)}{\left[m_{1} v+m_{2} x_{1}^{*}\left(w x_{1}^{*}-c\right)\right]\left[m_{1} v+m_{2} Q_{1}\left(w x_{1}^{*}-c\right)\right]} \mathrm{d} s \\
& +\frac{a_{2} x_{2}^{*}}{k+Q_{1}} \int_{t-\tau_{2}}^{t}\left[\frac{a_{2} P_{2} x_{1}^{*}\left(e^{y_{1}\left(t-\tau_{2}\right)}-1\right)}{\left(k+x_{1}^{*}\right)\left(k+Q_{1}\right)}-\frac{a_{2}\left(k+x_{1}^{*}\right) Q_{2}\left(e^{y_{2}\left(t-\tau_{2}\right)}-1\right)}{\left(k+P_{1}\right)}\right] \mathrm{d} s,
\end{aligned}
$$

where $P_{i}, Q_{i}(i=1,2)$ have been defined in Lemma 2 .

Furthermore, let

$$
\begin{aligned}
W_{4}(t)= & W_{3}(t)+\frac{a_{2} k x_{2}^{*} P_{1}}{\left(k+x_{1}^{*}\right)\left(k+P_{1}\right)} \int_{t-\tau_{2}}^{t} \int_{z}^{t} b_{1}\left|e^{y_{1}\left(s-\tau_{1}\right)}-1\right| \mathrm{d} s \mathrm{~d} z+\frac{a_{2} k x_{2}^{*} P_{1}}{\left(k+x_{1}^{*}\right)\left(k+P_{1}\right)} \int_{t-\tau_{2}}^{t} \int_{z}^{t} \frac{a_{1} x_{2}^{*} k}{x_{1}^{*}\left(k+x_{1}^{*}\right)\left(k+Q_{1}\right)}\left|e^{y_{2}(s)}-1\right| \mathrm{d} s \mathrm{~d} z \\
& -\frac{a_{2} k x_{2}^{*} P_{1}}{\left(k+x_{1}^{*}\right)\left(k+P_{1}\right)} \int_{t-\tau_{2}}^{t} \int_{z}^{t} \frac{q v m_{2}\left(w P_{1}-c\right)}{\left[m_{1} v+m_{2} x_{1}^{*}\left(w x_{1}^{*}-c\right)\right]\left[m_{1} v+m_{2} Q_{1}\left(w x_{1}^{*}-c\right)\right]}-1 \mid \mathrm{d} s \mathrm{~d} z \\
& +\frac{a_{2} x_{2}^{*}}{k+Q_{1}} \int_{t-\tau_{2}}^{t} \int_{z}^{t} \frac{a_{2} P_{2} x_{1}^{*}}{\left(k+x_{1}^{*}\right)\left(k+Q_{1}\right)}\left|e^{y_{1}\left(s-\tau_{2}\right)}-1\right| \mathrm{d} s \mathrm{~d} z-\frac{a_{2} x_{2}^{*}}{k+Q_{1}} \int_{t-\tau_{2}}^{t} \int_{z}^{t} \frac{a_{2}\left(k+x_{1}^{*}\right) Q_{2}}{\left(k+P_{1}\right)}\left|e^{y_{2}\left(s-\tau_{2}\right)}-1\right| \mathrm{d} \mathrm{d} z
\end{aligned}
$$

By virtue of (A.1) and computing the upper right derivative of $W_{4}(t)$ along the solution of system (24), it can be obtained that 


$$
\begin{aligned}
D^{+} W_{4}(t) \leq & D^{+} W_{3}(t)+\frac{a_{2} k b_{1} x_{2}^{*} P_{1} \tau_{2}}{\left(k+x_{1}^{*}\right)\left(k+P_{1}\right)}\left|e^{y_{1}\left(t-\tau_{1}\right)}-1\right|+\frac{a_{1} a_{2} k^{2} x_{2}^{* 2} P_{1} \tau_{2}}{x_{1}^{*}\left(k+x_{1}^{*}\right)^{2}\left(k+P_{1}\right)\left(k+Q_{1}\right)}\left|e^{y_{2}(t)}-1\right| \\
& -\frac{a_{2} k x_{2}^{*} q v m_{2} P_{1}\left(w P_{1}-c\right) \tau_{2}}{\left(k+x_{1}^{*}\right)\left(k+P_{1}\right)\left[m_{1} v+m_{2} x_{1}^{*}\left(w x_{1}^{*}-c\right)\right]\left[m_{1} v+m_{2} Q_{1}\left(w x_{1}^{*}-c\right)\right]}\left|e^{y_{2}(t)}-1\right| \\
& +\frac{a_{2}^{2} x_{2}^{*} \tau_{2}}{k+Q_{1}}\left[\frac{P_{2} x_{1}^{*}}{\left(k+x_{1}^{*}\right)\left(k+Q_{1}\right)}-\frac{\left(k+x_{1}^{*}\right) Q_{2}}{\left(k+P_{1}\right)}\right]\left|e^{y_{2}\left(t-\tau_{2}\right)}-1\right|-\frac{a_{2} x_{1}^{*} x_{2}^{*}}{\left(k+x_{1}^{*}\right)\left(k+P_{1}\right)} \int_{t-\tau_{2}}^{t} \\
& \cdot\left[b_{1} P_{1}\left(e^{y_{1}\left(s-\tau_{1}\right)}-1\right)+\frac{a_{1} x_{2}^{*} k P_{1}\left(e^{y_{2}(s)}-1\right)}{x_{1}^{*}\left(k+x_{1}^{*}\right)\left(k+Q_{1}\right)}\right] \mathrm{d} s+\frac{a_{2} x_{1}^{*} x_{2}^{*}}{\left(k+x_{1}^{*}\right)\left(k+P_{1}\right)} \int_{t-\tau_{2}}^{t} \frac{q v m_{2} P_{1}\left(w P_{1}(t)-c\right)\left(e^{y_{2}(s)}-1\right)}{\left[m_{1} v+m_{2} x_{1}^{*}\left(w x_{1}^{*}-c\right)\right]\left[m_{1} v+m_{2} Q_{1}\left(w x_{1}^{*}-c\right)\right]} \mathrm{d} s \\
& -\frac{a_{2} x_{2}^{*}}{k+Q_{1}} \int_{t-\tau_{2}}^{t}\left[\frac{a_{2} P_{2} x_{1}^{*}\left(e^{y_{1}\left(t-\tau_{2}\right)}-1\right)}{\left(k+x_{1}^{*}\right)\left(k+Q_{1}\right)}-\frac{a_{2}\left(k+x_{1}^{*}\right) Q_{2}\left(e^{y_{2}\left(t-\tau_{2}\right)}-1\right)}{\left(k+P_{1}\right)}\right] \mathrm{d} .
\end{aligned}
$$

By defining $W(t)=W_{2}(t)+W_{4}(t)$, it is easy to show that $W(t)>\left|y_{1}(t)\right|+\left|y_{2}(t)\right|$. By virtue of (A.3), (A.6), and simple computations, it can be obtained that

$$
D^{+} W(t) \leq-S_{1}\left(\tau_{1}, \tau_{2}\right)\left|e^{y_{1}(t)}-1\right|-S_{2}\left(\tau_{1}, \tau_{2}\right)\left|e^{y_{2}(t)}-1\right|
$$

where $S_{i}\left(\tau_{1}, \tau_{2}\right), i=1,2$ are defined as follows:

$$
\begin{aligned}
S_{1}\left(\tau_{1}, \tau_{2}\right)= & \frac{b_{1} P_{1}\left(x_{1}^{*}+Q_{1} \tilde{W}\right)}{Q_{1}}-\frac{q v m_{2}\left[x_{1}^{*}\left(w x_{1}^{*}-c\right)+P_{1} \tilde{W} \tau_{1}\left(w P_{1}-c\right)\right]}{\left[m_{1} v+m_{2} x_{1}^{*}\left(w x_{1}^{*}-c\right)\right]\left[m_{1} v+m_{2} Q_{1}\left(w x_{1}^{*}-c\right)\right]}-\frac{a_{2} k b_{1} x_{2}^{*} P_{1} \tau_{2}}{\left(k+x_{1}^{*}\right)\left(k+P_{1}\right)}, \\
S_{2}\left(\tau_{1}, \tau_{2}\right)= & \frac{a_{1} k x_{2}^{*}\left(\tilde{W} \tau_{1}+x_{1}^{*}\right)}{x_{1}^{*}\left(k+x_{1}^{*}\right)\left(k+Q_{1}\right)}-\frac{a_{1} a_{2} k^{2} x_{2}^{* 2} P_{1} \tau_{2}}{x_{1}^{*}\left(k+x_{1}^{*}\right)^{2}\left(k+P_{1}\right)\left(k+Q_{1}\right)}-\frac{a_{2}^{2} x_{2}^{*} \tau_{2}}{k+Q_{1}}\left[\frac{P_{2} x_{1}^{*}}{\left(k+x_{1}^{*}\right)\left(k+Q_{1}\right)}-\frac{\left(k+x_{1}^{*}\right) Q_{2}}{\left(k+P_{1}\right)}\right] \\
& +\frac{a_{2} k x_{2}^{*} q v m_{2} P_{1}\left(w P_{1}-c\right) \tau_{2}}{\left(k+x_{1}^{*}\right)\left(k+P_{1}\right)\left[m_{1} v+m_{2} x_{1}^{*}\left(w x_{1}^{*}-c\right)\right]\left[m_{1} v+m_{2} Q_{1}\left(w x_{1}^{*}-c\right)\right]},
\end{aligned}
$$

where $P_{i}, Q_{i}(i=1,2)$ have been defined in Lemma 2 and $\tilde{W}_{1}$ has been defined in (A.3).

By using the mean value theorem [35], for $\vartheta_{1}(t) \in(0,1)$, $\vartheta_{2}(t) \in(0,1)$, and $\vartheta_{1}(t) \neq \vartheta_{2}(t)$ it can be obtained that

$$
\begin{aligned}
D^{+} W(t) & \leq-S_{1}\left(\tau_{1}, \tau_{2}\right) e^{\vartheta_{1}(t)}\left|y_{1}(t)\right|-S_{2}\left(\tau_{1}, \tau_{2}\right) e^{\vartheta_{2}(t)}\left|y_{2}(t)\right| \\
& \leq-S_{1}\left(\tau_{1}, \tau_{2}\right)\left|y_{1}(t)\right|-S_{2}\left(\tau_{1}, \tau_{2}\right)\left|y_{2}(t)\right| \\
& \leq-\min \left\{S_{1}\left(\tau_{1}, \tau_{2}\right), S_{2}\left(\tau_{1}, \tau_{2}\right)\right\}\left\{\left|y_{1}(t)\right|+\left|y_{2}(t)\right|\right\} .
\end{aligned}
$$

If $\min \left\{S_{1}\left(\tau_{1}, \tau_{2}\right), S_{2}\left(\tau_{1}, \tau_{2}\right)\right\}>0$, then it is easy to derive that $\left(\tau_{1}, \tau_{2}, v\right) \in H_{2}$ and $H_{2}$ is as follows:

$$
H_{2}=\left\{\left(\tau_{1}, \tau_{2}, v\right) \mid 0<\tau_{1}<\tau_{1}^{*}, 0<\tau_{2}<\tau_{2}^{*}, v>0\right\},
$$

where $\tau_{1}^{*}, \tau_{2}^{*}$ satisfies the following inequalities $S_{1}\left(\tau_{1}^{*}, \tau_{2}^{*}\right)>0$ and $S_{2}\left(\tau_{1}^{*}, \tau_{2}^{*}\right)>0$ hold.

\section{B. Proof of Theorem 2}

Proof 9. For system (25), it is easy to show that Lipschitz conditions hold. Hence, there exists a unique local solution $\left(x_{1}(t), x_{2}(t)\right)$ for $t \in\left[-\tau_{m}, \widehat{\tau}\right)$, where $\tau_{m}=\max \left\{\tau_{1}, \tau_{2}\right\}$ and $\widehat{\tau}$ represents the explosion time [38].

Subsequently, we will show that $\widehat{\tau}=\infty$, which implies that solution $\left(x_{1}(t), x_{2}(t)\right)$ is global, by assuming that $j_{0} \geq 1$ is sufficiently large such that $x_{1}(t) \in\left[\left(1 / j_{0}\right), j_{0}\right]$ and $x_{2}(t) \in\left[\left(1 / j_{0}\right), j_{0}\right]$ for $t \in\left[-\tau_{m}, 0\right]$. With the purpose of facilitating the following analysis, the stopping time [38] for any $j \geq j_{0}$ is defined as follows:

$$
t_{j}=\inf \left\{t \in\left[-\tau_{m}, \widehat{\tau}\right) \mid x_{1}(t) \notin\left(\frac{1}{j}, j\right), x_{2}(t) \notin\left(\frac{1}{j}, j\right)\right\} .
$$

Let $\varnothing$ represent an empty set and $\inf \varnothing=\infty$. It is easy to show that $t_{j}$ increases as $j$ increases through $\infty$, by defining $\tau_{\infty}=\lim _{j \rightarrow \infty} t_{j}$, which derives that $\tau_{\infty} \geq \widehat{\tau}$ almost surely. Hence, if $\tau_{\infty}=\infty$ almost surely, then we can show $\widehat{\tau}=\infty$ and the solution $\left(x_{1}(t), x_{2}(t)\right) \in \mathbb{R}_{+}^{2}$ for all $t>0$ almost surely.

If $\tau_{\infty} \neq \infty$, then there exists a pair of constants $\tilde{T}>0$ and $0<\zeta<1$ such that $\mathbb{P}\left\{\tau_{\infty} \leq \tilde{T}\right\}>\zeta$. Hence, there exists some $j_{1} \geq j_{0}$, and $\mathbb{P}\left\{t_{j} \leq \tilde{T}\right\} \geq \zeta$. holds for all $j \geq j_{1}$.

Define a $\mathbb{C}^{2}$-function $\tilde{V}: \mathbb{R}_{+}^{2} \rightarrow \mathbb{R}_{+}$as follows: 


$$
\begin{aligned}
\tilde{V}\left(x_{1}(t), x_{2}(t)\right)= & x_{1}(t)-\frac{r}{b_{1}}-\frac{r}{b_{1}} \ln \frac{b_{1} x_{1}(t)}{r_{1}} \\
& +x_{2}(t)-1-\ln x_{2}(t) .
\end{aligned}
$$

By assuming that $\sigma_{j k}>0(j, k=1,2)$ are sufficiently small, by utilizing Lemma 2 of this paper and Itô's formula (5), it can be obtained that

$$
\begin{aligned}
\mathrm{d} \tilde{V}\left(x_{1}(t), x_{2}(t)\right)= & \left(1-\frac{r_{1}}{b_{1} x_{1}(t)}\right)\left[x_{1}\left(t-\tau_{1}\right)\left(r_{1}-b_{1} x_{1}\left(t-\tau_{1}\right)\right)-\frac{a_{1} x_{1}(t) x_{2}(t)}{k+x_{1}(t)}-\frac{q v x_{1}(t)}{m_{1} v+m_{2} x_{1}(t)\left(w x_{1}(t)-c\right)}\right] \mathrm{d} t \\
& +\left(1-\frac{1}{x_{2}(t)}\right)\left[x_{2}(t)\left(r_{2}-\frac{a_{2} x_{2}\left(t-\tau_{2}\right)}{k+x_{1}\left(t-\tau_{2}\right)}\right)\right] \mathrm{d} t+\frac{r_{1}}{2 b_{1}}\left(\sigma_{11}^{2}+\sigma_{21}^{2}+\frac{\sigma_{12}^{2}}{x_{1}^{2}(t)}+\frac{\sigma_{22}^{2}}{x_{2}^{2}(t)}\right) \mathrm{d} t \\
& +\left[\int_{\mathbb{V}} \frac{r_{1}}{b_{1}}\left[\gamma_{1}(u)-\ln \left(1+\gamma_{1}(u)\right)\right] \lambda \mathrm{d} u+\int_{\mathbb{V}}\left[\gamma_{2}(u)-\ln \left(1+\gamma_{2}(u)\right)\right] \lambda \mathrm{d} u\right] \mathrm{d} t \\
& +\left[\left(\left(x_{1}(t)-\frac{r_{1}}{b_{1}}\right) \sigma_{11}+\left(x_{2}(t)-1\right) \sigma_{21}\right) \xi_{1}(t)+\left(\frac{r_{1} \sigma_{11}}{b_{1}}+\sigma_{21}\right) \xi_{2}(t)\right] \mathrm{d} t \\
& +\int_{\mathbb{V}} \frac{r_{1}}{b_{1}}\left[\gamma_{1}(u) x_{1}(t-)-\ln \left(1+\gamma_{1}(u)\right)\right] \tilde{N}(\mathrm{~d} t, \mathrm{~d} u)+\int_{\mathbb{V}}\left[\gamma_{2}(u) x_{2}(t-)-\ln \left(1+\gamma_{2}(u)\right)\right] \tilde{N}(\mathrm{~d} t, \mathrm{~d} u) .
\end{aligned}
$$

In order to facilitate the following analysis, $J$ is defined as follows:

$$
\begin{aligned}
J= & \frac{r_{1}\left[Q_{1}+4 P_{1}\left(b_{1}-r_{1}\right)\right]}{4 b_{1} Q_{1}}+\frac{r_{1}-b Q_{1}}{b}\left(\frac{q}{m_{1}}+\frac{a_{1} P_{2}}{k}\right) \\
& +\left(P_{2}-1\right)\left(r_{2}+a_{2}\right)+\frac{r_{1}}{2 b_{1}}\left(\sigma_{11}^{2}+\sigma_{21}^{2}+\frac{\sigma_{12}^{2}}{Q_{1}^{2}}+\frac{\sigma_{22}^{2}}{Q_{1}^{2}}\right) \\
& +\int_{\mathbb{V}} \frac{r_{1}}{b_{1}}\left[\gamma_{1}(u)-\ln \left(1+\gamma_{1}(u)\right)\right] \lambda \mathrm{d} u \\
& +\int_{\mathbb{V}}\left[\gamma_{2}(u)-\ln \left(1+\gamma_{2}(u)\right)\right] \lambda \mathrm{d} u,
\end{aligned}
$$

where $P_{i}, Q_{i}(i=1,2)$ are defined in Lemma 2.

If $\int_{\mathbb{Y}}\left[\gamma_{i}(u)-\ln \left(1+\gamma_{i}(u)\right)\right] \lambda \mathrm{d} u \leq \tilde{\gamma}_{i}(i=1,2)$ and $\tilde{\gamma}_{i}$ are positive constants, then

$$
\begin{aligned}
J \leq & \frac{r_{1}\left[Q_{1}+4 P_{1}\left(b_{1}-r_{1}\right)\right]}{4 b_{1} Q_{1}}+\frac{r_{1}-b Q_{1}}{b}\left(\frac{q}{m_{1}}+\frac{a_{1} P_{2}}{k}\right) \\
& +\left(P_{2}-1\right)\left(r_{2}+a_{2}\right)+\frac{r_{1}}{2 b_{1}}\left(\sigma_{11}^{2}+\sigma_{21}^{2}+\frac{\sigma_{12}^{2}}{Q_{1}^{2}}+\frac{\sigma_{22}^{2}}{Q_{1}^{2}}\right) \\
& +\frac{r_{1}}{b_{1}} \tilde{\gamma}_{1}+\tilde{\gamma}_{2} .
\end{aligned}
$$

Consequently, it follows from (B.5) that

$$
\begin{aligned}
\mathrm{d} \tilde{V}\left(x_{1}(t), x_{2}(t)\right) \leq & {\left[J+\left(\left(x_{1}(t)-\frac{r_{1}}{b_{1}}\right) \sigma_{11}+\left(x_{2}(t)-1\right) \sigma_{21}\right) \xi_{1}(t)\right.} \\
& \left.+\left(\frac{r_{1} \sigma_{11}}{b_{1}}+\sigma_{21}\right) \xi_{2}(t)\right] \mathrm{d} t \\
& +\int_{\mathbb{Y}} \frac{r_{1}}{b_{1}}\left[\gamma_{1}(u) x_{1}(t-)-\ln \left(1+\gamma_{1}(u)\right)\right] \tilde{N}(\mathrm{~d} t, \mathrm{~d} u) \\
& +\int_{\mathbb{V}}\left[\gamma_{2}(u) x_{2}(t-)-\ln \left(1+\gamma_{2}(u)\right)\right] \tilde{N}(\mathrm{~d} t, \mathrm{~d} u) .
\end{aligned}
$$

By integrating both sides of (B.6) from 0 to $t_{j} \wedge \tilde{T}=\min$ $\left\{t_{j}, \tilde{T}\right\}, \tilde{T}$ has been defined in (25) and then expectations can be computed as follows:

$$
\mathbb{E} \tilde{V}\left(x_{1}\left(t_{j} \wedge \tilde{T}\right), x_{2}\left(t_{j} \wedge \tilde{T}\right)\right) \leq \tilde{V}\left(x_{1}(0), x_{2}(0)\right)+J \mathbb{E}\left(t_{j} \wedge \tilde{T}\right),
$$

which follows that

$$
\mathbb{E} \tilde{V}\left(x_{1}\left(t_{j} \wedge \tilde{T}\right), x_{2}\left(t_{j} \wedge \tilde{T}\right)\right) \leq \tilde{V}\left(x_{1}(0), x_{2}(0)\right)+J \tilde{T} .
$$

When $j \geq j_{1}$, we define $\Omega_{j}=\left\{t_{j} \leq \tilde{T}\right\}$; it is easy to derive that $\mathbb{P}\left(\Omega_{i}\right) \geq \zeta$ based on the fact $\mathbb{P}\left\{t_{j} \leq \tilde{T}\right\} \geq \zeta$. holds for all $j \geq j_{1}$. Furthermore, it can be obtained that $x_{1}\left(t_{j}, \varepsilon\right)$ or $x_{2}\left(t_{j}, \varepsilon\right)$ equals to either $j$ or $1 / j$ which holds for any $\varepsilon \in \Omega_{j}$, which follows that $\tilde{V}\left(x_{1}\left(t_{j}, \varepsilon\right), x_{2}\left(t_{j}, \varepsilon\right)\right)$ is no less than either $j-1-\ln j$ or $(1 / j)-1-\ln (1 / j)$.

Consequently, it derives that

$$
\tilde{V}\left(x_{1}\left(t_{j}, \varepsilon\right), x_{2}\left(t_{j}, \varepsilon\right)\right) \geq[j-1-\ln j] \wedge\left[\frac{1}{j}-1-\ln \frac{1}{j}\right] .
$$

It follows from (B.8) that

$$
\begin{aligned}
\tilde{V}\left(x_{1}(0), x_{2}(0)\right)+J \tilde{T} & \geq \mathbb{E}\left[I_{\Omega_{j}}(\varepsilon) \tilde{V}\left(x_{1}\left(t_{j}, \varepsilon\right), x_{2}\left(t_{j}, \varepsilon\right)\right)\right] \\
& \geq[j-1-\ln j] \wedge\left[\frac{1}{j}-1-\ln \frac{1}{j}\right],
\end{aligned}
$$


where $I_{\Omega_{j}}$ represents indicator function of $\Omega_{j}$. When $j \rightarrow \infty$, it derives that

$$
\infty=\tilde{V}\left(x_{1}(0), x_{2}(0)\right)+J \tilde{T}<\infty
$$

which is a contradiction.

Based on the above analysis, it can be obtained that $\tau_{\infty}=\infty$ and $\left(x_{1}(t), x_{2}(t)\right)$ will not explode in a finite time almost surely.

\section{Proof of Theorem 5}

Proof 10. Firstly, we construct the following function:

$$
W_{11}(t)=x_{1}\left(t+\tau_{1}\right)-x_{1}^{*}-x_{1}^{*} \ln \frac{x_{1}\left(t+\tau_{1}\right)}{x_{1}^{*}}
$$

By using simple computations, it can be obtained that

$$
\begin{aligned}
& \mathrm{d} W_{11}(t)=\left(x_{1}\left(t+\tau_{1}\right)-x_{1}^{*}\right) \\
& {\left[\frac{b_{1} x_{1}(t)}{x_{1}\left(t+\tau_{1}\right)}\left(x_{1}^{*}-x_{1}(t)\right)+a_{1}\right.} \\
& \left.\left(\frac{x_{2}^{*} x_{1}(t)}{x_{1}\left(t+\tau_{1}\right)\left(k+x_{1}^{*}\right)}-\frac{x_{2}\left(t+\tau_{1}\right)}{k+x_{1}\left(t+\tau_{1}\right)}\right)\right] \mathrm{d} t \\
& +q v\left(x_{1}\left(t+\tau_{1}\right)-x_{1}^{*}\right) \\
& \cdot\left[\frac{x_{1}(t)}{x_{1}\left(t+\tau_{1}\right)\left[m_{1} v+m_{2} x_{1}^{*}\left(w x_{1}^{*}-c\right)\right]}\right. \\
& \left.-\frac{1}{m_{1} v+m_{2} x_{1}\left(t+\tau_{1}\right)\left(w x_{1}^{*}-c\right)}\right] \mathrm{d} t \\
& +\left[\frac{x_{1}^{*}}{2}\left(\sigma_{11}^{2}+\frac{\sigma_{12}^{2}}{x_{1}^{2}(t)}\right)\right. \\
& \left.+\int_{\mho} x_{1}^{*}\left[\gamma_{1}(u)-\ln \left(1+\gamma_{1}(u)\right)\right] \lambda \mathrm{d} u\right] \mathrm{d} t \\
& +\left[\left(x_{1}(t)-x_{1}^{*}\right) \sigma_{11} \xi_{1}(t)+x_{1}^{*} \sigma_{12} \xi_{2}(t)\right] \mathrm{d} t \\
& +\int_{\mathbb{Y}} x_{1}^{*}\left[\gamma_{1}(u) x_{1}(t-)-\ln \left(1+\gamma_{1}(u)\right)\right] \tilde{N}(\mathrm{~d} t, \mathrm{~d} u) \\
& =\frac{x_{1}(t)\left(x_{1}\left(t+\tau_{1}\right)-x_{1}^{*}\right)}{x_{1}\left(t+\tau_{1}\right)} \\
& \cdot\left[b_{1}\left(x_{1}^{*}-x_{1}(t)\right)+\frac{a_{1} x_{2}^{*}}{k+x_{1}^{*}}+\frac{q v}{m_{1} v+m_{2} x_{1}^{*}\left(w x_{1}^{*}-c\right)}\right] \mathrm{d} t \\
& -\left(x_{1}\left(t+\tau_{1}\right)-x_{1}^{*}\right) \\
& \cdot\left[\frac{a_{1} x_{2}\left(t+\tau_{1}\right)}{k+x_{1}\left(t+\tau_{1}\right)}+\frac{q v}{m_{1} v+m_{2} x_{1}\left(t+\tau_{1}\right)\left(w x_{1}^{*}-c\right)}\right] \mathrm{d} t \\
& +\left[\frac{x_{1}^{*}}{2}\left(\sigma_{11}^{2}+\frac{\sigma_{12}^{2}}{x_{1}^{2}(t)}\right)+\int_{\mathbb{Y}} x_{1}^{*}\left[\gamma_{1}(u)-\ln \left(1+\gamma_{1}(u)\right)\right] \lambda \mathrm{d} u\right] \mathrm{d} t \\
& +\left[\left(x_{1}(t)-x_{1}^{*}\right) \sigma_{11} \xi_{1}(t)+x_{1}^{*} \sigma_{12} \xi_{2}(t)\right] \mathrm{d} t \\
& +\int_{\mathbb{Y}} x_{1}^{*}\left[\gamma_{1}(u) x_{1}(t-)-\ln \left(1+\gamma_{1}(u)\right)\right] \tilde{N}(\mathrm{~d} t, \mathrm{~d} u) \\
& =\left[\mathscr{L} W_{11}+\left(x_{1}(t)-x_{1}^{*}\right) \sigma_{11} \xi_{1}(t)+x_{1}^{*} \sigma_{12} \xi_{2}(t)\right] \mathrm{d} t \\
& +\int_{\mho} x_{1}^{*}\left[\gamma_{1}(u) x_{1}(t-)-\ln \left(1+\gamma_{1}(u)\right)\right] \tilde{N}(\mathrm{~d} t, \mathrm{~d} u),
\end{aligned}
$$

where $\mathscr{L} W_{11}$ satisfies the following inequalities:

$$
\begin{aligned}
& \mathscr{L} W_{11} \leq b_{1} x_{1}(t)\left(x_{1}^{*}-x_{1}(t)\right)+x_{1}(t) \\
& \cdot\left[\frac{a_{1} x_{2}^{*}}{k+x_{1}^{*}}+\frac{q v}{m_{1} v+m_{2} x_{1}^{*}\left(w x_{1}^{*}-c\right)}\right] \\
& -\frac{a_{1} x_{1}\left(t+\tau_{1}\right) x_{2}\left(t+\tau_{1}\right)}{k+x_{1}\left(t+\tau_{1}\right)} \\
& -\frac{q v x_{1}\left(t+\tau_{1}\right)}{m_{1} v+m_{2}\left(w x_{1}^{*}-c\right) x_{1}\left(t+\tau_{1}\right)}-\frac{x_{1}^{*} x_{1}(t)}{x_{1}\left(t+\tau_{1}\right)} \\
& \cdot\left[b_{1}\left(x_{1}^{*}-x_{1}(t)\right)+\frac{a_{1} x_{2}^{*}}{k+x_{1}^{*}}+\frac{q v}{m_{1} v+m_{2} x_{1}^{*}\left(w x_{1}^{*}-c\right)}\right] \\
& +x_{1}^{*}\left[\frac{a_{1} x_{2}\left(t+\tau_{1}\right)}{k+x_{1}\left(t+\tau_{1}\right)}+\frac{q v}{m_{1} v+m_{2} x_{1}\left(t+\tau_{1}\right)\left(w x_{1}^{*}-c\right)}\right] \\
& +\frac{x_{1}^{*}}{2}\left(\sigma_{11}^{2}+\frac{\sigma_{12}^{2}}{x_{1}^{2}(t)}\right)+\int_{\Upsilon} x_{1}^{*}\left[\gamma_{1}(u)-\ln \left(1+\gamma_{1}(u)\right)\right] \lambda \mathrm{d} u \\
& \leq-b_{1}\left(x_{1}(t)-x_{1}^{*}\right)^{2}+x_{1}(t) \\
& \cdot\left[\frac{a_{1} x_{2}^{*}}{k+x_{1}^{*}}+\frac{q v}{m_{1} v+m_{2} x_{1}^{*}\left(w x_{1}^{*}-c\right)}\right] \\
& +x_{1}^{*}\left(\frac{a_{1} x_{2}\left(t+\tau_{1}\right)}{k}+\frac{q}{m_{1}}\right)+b_{1} x_{1}^{*}\left(x_{1}^{*}-x_{1}(t)\right) \\
& \cdot\left(1-\frac{x_{1}(t)}{x_{1}\left(t+\tau_{1}\right)}\right)-x_{1}^{*}\left(1+\ln \frac{x_{1}(t)}{x_{1}\left(t+\tau_{1}\right)}\right) \\
& \cdot\left[\frac{a_{1} x_{2}^{*}}{k+x_{1}^{*}}+\frac{q v}{m_{1} v+m_{2} x_{1}^{*}\left(w x_{1}^{*}-c\right)}\right] \\
& +\frac{x_{1}^{*}}{2}\left(\sigma_{11}^{2}+\frac{\sigma_{12}^{2}}{x_{1}^{2}(t)}\right)+\int_{\mathbb{Y}} x_{1}^{*}\left[\gamma_{1}(u)-\ln \left(1+\gamma_{1}(u)\right)\right] \lambda \mathrm{d} u \\
& \leq-b_{1}\left(x_{1}(t)-x_{1}^{*}\right)^{2}+x_{1}(t)\left[\frac{a_{1} x_{2}^{*}}{k+x_{1}^{*}}+\frac{q v}{m_{1} v+m_{2} x_{1}^{*}\left(w x_{1}^{*}-c\right)}\right] \\
& +x_{1}^{*}\left(\frac{a_{1} x_{2}\left(t+\tau_{1}\right)}{k}+\frac{q}{m_{1}}\right)+b_{1} x_{1}^{*}\left(x_{1}^{*}+\frac{x_{1}^{2}(t)}{x_{1}\left(t+\tau_{1}\right)}\right) \\
& -x_{1}^{*}\left(\ln \frac{x_{1}(t)}{x_{1}^{*}}-\ln \frac{x_{1}\left(t+\tau_{1}\right)}{x_{1}^{*}}+1\right) \\
& \cdot\left[\frac{a_{1} x_{2}^{*}}{k+x_{1}^{*}}+\frac{q v}{m_{1} v+m_{2} x_{1}^{*}\left(w x_{1}^{*}-c\right)}\right] \\
& +\frac{x_{1}^{*}}{2}\left(\sigma_{11}^{2}+\frac{\sigma_{12}^{2}}{x_{1}^{2}(t)}\right)+\int_{\curlyvee} x_{1}^{*}\left[\gamma_{1}(u)-\ln \left(1+\gamma_{1}(u)\right)\right] \lambda \mathrm{d} u .
\end{aligned}
$$

Based on (C.3), $W_{12}(t)$ is defined as follows,

$$
\begin{aligned}
W_{12}(t)= & W_{11}(t)-\frac{a x_{1}^{*}}{k} \int_{t}^{t+\tau_{1}} x_{2}(s) \mathrm{d} s+\frac{a_{1} x_{1}^{*} x_{2}^{*}}{k+x_{1}^{*}} \int_{t}^{t+\tau_{1}} \\
& \cdot\left[\ln \frac{x_{1}(s)}{x_{1}^{*}}-\ln \frac{x_{1}(s)}{x_{1}^{*}}+1\right] \mathrm{d} s \\
& +\frac{q v x_{1}^{*}}{m_{1} v+m_{2} x_{1}^{*}\left(w x_{1}^{*}-c\right)} \int_{t}^{t+\tau_{1}} \\
& \cdot\left[\ln \frac{x_{1}(s)}{x_{1}^{*}}-\ln \frac{x_{1}(s)}{x_{1}^{*}}+1\right] \mathrm{d} s .
\end{aligned}
$$

By using simple computations, it can be obtained that 


$$
\begin{aligned}
\mathscr{L} W_{12} \leq & -b_{1}\left(x_{1}(t)-x_{1}^{*}\right)^{2}+x_{1}(t) \\
& \cdot\left[\frac{a_{1} x_{2}^{*}}{k+x_{1}^{*}}+\frac{q v}{m_{1} v+m_{2} x_{1}^{*}\left(w x_{1}^{*}-c\right)}\right] \\
& +x_{1}^{*}\left(\frac{a_{1} x_{2}\left(t+\tau_{1}\right)}{k}+\frac{q}{m_{1}}\right) \\
& +b_{1} x_{1}^{*}\left(x_{1}^{*}+\frac{x_{1}^{2}(t)}{x_{1}\left(t+\tau_{1}\right)}\right)+\frac{x_{1}^{*}}{2}\left(\sigma_{11}^{2}+\frac{\sigma_{12}^{2}}{x_{1}^{2}(t)}\right) \\
& +\int_{\mathbb{Y}} x_{1}^{*}\left[\gamma_{1}(u)-\ln \left(1+\gamma_{1}(u)\right)\right] \lambda \mathrm{d} u .
\end{aligned}
$$

Secondly, we construct the following function:

$$
W_{13}(t)=\frac{\left[x_{1}\left(t+\tau_{1}\right)-x_{1}^{*}\right]^{2}}{2} .
$$

By using similar arguments in (C.3) and (C.5), it follows from simple computations that

$$
\begin{aligned}
\mathscr{L} W_{13}= & b_{1}\left(x_{1}^{*}-x_{1}\left(t+\tau_{1}\right)\right)\left(x_{1}(t)-x_{1}^{*}\right)^{2}+b_{1} x_{1}^{*}\left(x_{1}^{*}-x_{1}\left(t+\tau_{1}\right)\right)\left(x_{1}(t)-x_{1}^{*}\right)+a_{1}\left(x_{1}\left(t+\tau_{1}\right)-x_{1}^{*}\right)\left[\frac{x_{2}^{*} x_{1}(t)}{k+x_{1}^{*}}-\frac{x_{1}\left(t+\tau_{1}\right) x_{2}\left(t+\tau_{1}\right)}{k+x_{1}\left(t+\tau_{1}\right)}\right] \\
& +q v\left(x_{1}\left(t+\tau_{1}\right)-x_{1}^{*}\right)\left[\frac{x_{1}(t)}{m_{1} v+\left(w_{1} x_{1}^{*}-c\right) m_{2} x_{1}^{*}}-\frac{\sigma_{1}^{2}}{m_{1} v+m_{2} x_{1}\left(t+\tau_{1}\right)\left(w x_{1}\left(t+\tau_{1}\right)-c\right)}\right]+\sigma_{11}^{2} x_{1}^{* 2}+\frac{\sigma_{12}^{2}}{2} \\
& +\int_{\mathbb{Y}}\left[\gamma_{1}(u)-\ln \left(1+\gamma_{1}(u)\right)\right] \lambda \mathrm{d} u \leq b_{1} x_{1}^{*}\left(x_{1}(t)-x_{1}^{*}\right)^{2}-b_{1} x_{1}^{*}\left(x_{1}-x_{1}^{*}\right)\left[x_{1}\left(t+\tau_{1}\right)-x_{1}(t)+x_{1}(t)-x_{1}^{*}\right] \\
& +a_{1}\left(x_{1}\left(t+\tau_{1}\right)-x_{1}^{*}\right) \frac{x_{2}^{*} x_{1}(t)\left[k+x_{1}\left(t+\tau_{1}\right)\right]-\left(k+x_{1}^{*}\right)}{\left(k+x_{1}^{*}\right)\left(k+x_{1}\left(t+\tau_{1}\right)\right)}+q v\left(x_{1}\left(t+\tau_{1}\right)-x_{1}^{*}\right) \\
& +\left[\frac{x_{1}\left(t+\tau_{1}\right)}{m_{1} v+\left(w_{1} x_{1}^{*}-c\right) m_{2} x_{1}^{*}}-\frac{x_{1}}{m_{1} v+m_{2} x_{1}\left(t+\tau_{1}\right)\left(w x_{1}\left(t+\tau_{1}\right)-c\right)}\right]+\sigma_{11}^{2} x_{1}^{* 2}+\frac{\sigma_{12}^{2}}{2} \\
& +\int_{\mathbb{Y}}\left[\gamma_{1}(u)-\ln \left(1+\gamma_{1}(u)\right)\right] \lambda \mathrm{d} u \leq b_{1} x_{1}^{* 2}\left(x_{1}(t)+x_{1}\left(t+\tau_{1}\right)\right)+a_{1} x_{1}\left(t+\tau_{1}\right)\left[\frac{x_{2}^{*} x_{1}(t)}{k+x_{1}^{*}}+\frac{x_{1}^{*} x_{2}\left(t+\tau_{1}\right)}{k+x_{1}\left(t+\tau_{1}\right)}\right] \\
& \left.+q v\left[\frac{x_{1}^{*} x_{1}\left(t+\tau_{1}\right)}{m_{1} v+m_{2} x_{1}^{*}\left(w x_{1}(t)-c\right)}+\frac{\sigma_{12}^{2}}{m_{1} v+m_{2} x_{1}\left(t+\tau_{1}\right)\left(w x_{1}\left(t+\tau_{1}\right)-c\right)}\right]+\sigma_{11}^{2} x_{1}^{* 2}+\frac{\sigma_{12}}{2}+\gamma_{\mathbb{Y}}(u)-\ln \left(1+\gamma_{1}(u)\right)\right] \lambda \mathrm{d} u .
\end{aligned}
$$

Let $W_{1}(t)=W_{12}(t)+W_{13}(t)$; it follows from (C.5) and (C.7) that

$$
\begin{aligned}
\mathscr{L} W_{1} \leq & -b_{1}\left(x_{1}(t)-x_{1}^{*}\right)^{2}+x_{1}(t)\left[\frac{a_{1} x_{2}^{*}}{k+x_{1}^{*}}+\frac{q v}{m_{1} v+m_{2} x_{1}^{*}\left(w x_{1}^{*}-c\right)}\right]+b_{1} x_{1}^{*}\left(x_{1}^{*}+\frac{x_{1}^{2}(t)}{x_{1}\left(t+\tau_{1}\right)}\right)+\frac{x_{1}^{*}}{2}\left(\sigma_{11}^{2}+\frac{\sigma_{12}^{2}}{x_{1}^{2}(t)}\right) \\
& +\int_{\mathbb{V}} x_{1}^{*}\left[\gamma_{1}(u)-\ln \left(1+\gamma_{1}(u)\right)\right] \lambda \mathrm{d} u+b_{1} x_{1}^{* 2}\left(x_{1}(t)+x_{1}\left(t+\tau_{1}\right)\right)+a_{1} x_{1}\left(t+\tau_{1}\right)\left[\frac{x_{2}^{*} x_{1}(t)}{k+x_{1}^{*}}+\frac{x_{1}^{*} x_{2}\left(t+\tau_{1}\right)}{k+x_{1}(t+\tau)}\right] \\
& +x_{1}^{*}\left(\frac{a_{1} x_{2}\left(t+\tau_{1}\right)}{k}+\frac{q}{m_{1}}\right)+q v\left[\frac{x_{1}(t) x_{1}\left(t+\tau_{1}\right)}{m_{1} v+m_{2} x_{1}^{*}\left(w x_{1}(t)-c\right)}+\frac{\left.x_{1}\right)}{m_{1} v+m_{2} x_{1}\left(t+\tau_{1}\right)\left(w x_{1}\left(t+\tau_{1}\right)-c\right)}\right] \\
& +\sigma_{11}^{2} x_{1}^{* 2}+\frac{\sigma_{12}^{2}}{2}+\int_{\mathbb{V}}\left[\gamma_{1}(u)-\ln \left(1+\gamma_{1}(u)\right)\right] \lambda \mathrm{d} u .
\end{aligned}
$$

If $\int_{\mathbb{Y}}\left[\gamma_{1}(u)-\ln \left(1+\gamma_{1}(u)\right)\right] \lambda \mathrm{d} u \leq \tilde{\gamma}_{1}$ and $\tilde{\gamma}_{1}$ are positive constants and $\sigma_{j 1}>0(j=1,2)$ is sufficiently small, then 


$$
\begin{aligned}
\mathscr{L} W_{1} \leq & -b_{1}\left(x_{1}(t)-x_{1}^{*}\right)^{2}+P_{1}\left(\frac{a_{1} x_{2}^{*}}{k+x_{1}^{*}}+\frac{q}{m_{1}}\right)+\frac{a_{1} P_{1}^{2}\left(x_{1}^{*}+x_{2}^{*}\right)}{k+x_{1}^{*}} \\
& +b_{1} x_{1}^{*}\left(x_{1}^{*}+\frac{P_{1}^{2}}{Q_{1}}\right)+x_{1}^{*}\left(\frac{a_{1} P_{2}}{k}+\frac{q}{m_{1}}\right) \\
& +q v\left[\frac{P_{1}^{2}}{m_{1} v+m_{2} x_{1}^{*}\left(w Q_{1}-c\right)}+\frac{x_{1}^{*}}{m_{2}\left(w Q_{1}-c\right)}\right] \\
& +\frac{x_{1}^{*}\left(1+x_{1}^{*}\right) \sigma_{11}^{2}}{2}+\frac{\left(2+Q_{1}^{2}\right) \sigma_{12}^{2}}{2 Q_{1}^{2}} \\
& +\left(1+x_{1}^{*}\right) \tilde{\gamma}_{1} \leq-b_{1}\left(x_{1}(t)-x_{1}^{*}\right)^{2} \\
& +\frac{a_{1} m_{1} P_{1}\left[x_{2}^{*}\left(1+P_{1}\right)+x_{1}^{*} P_{1}\right]+q P_{1}\left(k+x_{1}^{*}\right)}{m_{1}\left(k+x_{1}^{*}\right)}
\end{aligned}
$$$$
+\frac{b_{1} x_{1}^{*}\left(Q_{1} x_{1}^{*}+P_{1}^{2}\right)}{Q_{1}}+\frac{x_{1}^{*}\left(a_{1} m_{1} P_{2}+q k\right)}{k m_{1}}
$$

where $P_{1}, P_{2}, Q_{1}$ are defined in Lemma 2 of this paper.

By integrating both sides of (C.9) from 0 to $t$ and deriving expectation, it is easy to show that

$$
\begin{aligned}
\mathbb{E} W_{1}(t)-\mathbb{E} W_{1}(0) \leq & -b_{1} \mathbb{E} \int_{0}^{t}\left(x_{1}(s)-x_{1}^{*}\right)^{2} \mathrm{~d} s \\
& +\left\{\frac{a_{1} m_{1} P_{1}\left[x_{2}^{*}\left(1+P_{1}\right)+x_{1}^{*} P_{1}\right]+q P_{1}\left(k+x_{1}^{*}\right)}{m_{1}\left(k+x_{1}^{*}\right)}+\frac{b_{1} x_{1}^{*}\left(Q_{1} x_{1}^{*}+P_{1}^{2}\right)}{Q_{1}}+\frac{x_{1}^{*}\left(a_{1} m_{1} P_{2}+q k\right)}{k m_{1}}+\frac{q v\left(P_{1}^{2}+x_{1}^{* 2}\right)}{m_{2} x_{1}^{*}\left(w Q_{1}-c\right)}\right. \\
& \left.\quad+\frac{x_{1}^{*}\left(1+x_{1}^{*}\right) \sigma_{11}^{2}}{2}+\frac{\left(2+Q_{1}^{2}\right) \sigma_{12}^{2}}{2 Q_{1}^{2}}+\left(1+x_{1}^{*}\right) \tilde{\gamma}_{1}\right\} t
\end{aligned}
$$

It follows from (C.10) that

$$
\limsup _{t \rightarrow \infty} \frac{1}{t} \mathbb{E} \int_{0}^{t}\left[x_{1}(s)-x_{1}^{*}\right]^{2} \mathrm{~d} s \leq B_{1},
$$

where $B_{1}$ is defined as follows:

$$
\begin{aligned}
B_{1}= & \frac{a_{1} m_{1} k P_{1}\left[x_{1}^{*} P_{1}+x_{2}^{*}\left(1+P_{1}\right)\right]+\left(k+x_{1}^{*}\right)\left[q k\left(P+x_{1}^{*}\right)+a_{1} m_{1} P_{2} x_{1}^{*}\right]}{k m_{1} b_{1}\left(k+x_{1}^{*}\right)}+\frac{x_{1}^{*}\left(Q_{1} x_{1}^{*}+P_{1}^{2}\right)}{Q_{1}} \\
& +\frac{2 q v Q_{1}^{2}\left(P_{1}^{2}+x_{1}^{* 2}\right)+m_{2} x_{1}^{*}\left(w Q_{1}-c\right)\left[x_{1}^{*}\left(1+x_{1}^{*}\right) \sigma_{11}^{2}+\left(2+Q_{1}^{2}\right) \sigma_{12}^{2}+2 Q_{1}^{2}\left(1+x_{1}^{*}\right) \tilde{\gamma}_{1}\right]}{2 b_{1} m_{2} x_{1}^{*} Q_{1}^{2}\left(w Q_{1}-c\right)}
\end{aligned}
$$

Thirdly, we construct the following function:

$$
W_{21}(t)=x_{2}\left(t+\tau_{2}\right)-x_{2}^{*}-x_{2}^{*} \ln \frac{x_{2}\left(t+\tau_{2}\right)}{x_{2}^{*}}
$$

By using simple computations, it can be obtained that

$$
\begin{aligned}
\mathrm{d} W_{21}(t)=[ & a_{2}\left(x_{2}\left(t+\tau_{2}\right)-x_{2}^{*}\right)\left(\frac{x_{2}^{*}}{k+x_{1}^{*}}-\frac{x_{2}(t)}{k+x_{1}(t)}\right) \\
& \left.+\frac{x_{2}^{*} \sigma_{21}^{2} \sigma_{22}^{2}}{2}+\sigma_{21}\left(x_{1}\left(t+\tau_{2}\right)-x_{2}^{*}\right) \xi_{1}(t)\right] \mathrm{d} t
\end{aligned}
$$

$$
\begin{gathered}
+\left[\sigma_{22} \xi_{2}(t)+\int_{\mathbb{Y}}\left[\gamma_{2}(u)-\ln \left(1+\gamma_{2}(u)\right)\right] \lambda \mathrm{d} u\right] \mathrm{d} t \\
+\int_{\mathbb{Y}}\left[\gamma_{2}(u) x_{2}(t-)-\ln \left(1+\gamma_{2}(u)\right)\right] \tilde{N}(\mathrm{~d} t, \mathrm{~d} u) \\
=\left[\mathscr{L} W_{21}+\sigma_{21}\left(x_{1}\left(t+\tau_{2}\right)-x_{2}^{*}\right) \xi_{1}(t)+\sigma_{22} \xi_{2}(t)\right] \mathrm{d} t \\
+\int_{\mathbb{Y}}\left[\gamma_{2}(u) x_{2}(t-)-\ln \left(1+\gamma_{2}(u)\right)\right] \tilde{N}(\mathrm{~d} t, \mathrm{~d} u),
\end{gathered}
$$

where $\mathscr{L} W_{21}$ satisfies the following inequalities: 


$$
\begin{aligned}
\mathscr{L} W_{21}= & a_{2}\left(x_{2}\left(t+\tau_{2}\right)-x_{2}^{*}\right)\left[\frac{x_{2}^{*}-x_{2}(t)}{k+x_{1}^{*}}-\frac{x_{2}(t)\left(x_{1}(t)-x_{1}^{*}\right)}{\left(k+x_{1}^{*}\right)\left(k+x_{1}(t)\right)}\right] \\
& +\frac{x_{2}^{*} \sigma_{21}^{2} \sigma_{22}^{2}}{2}+\int_{\mathbb{Y}}\left[\gamma_{2}(u)-\ln \left(1+\gamma_{2}(u)\right)\right] \lambda \mathrm{d} u \\
\leq & -\frac{a_{2}\left(x_{2}\left(t+\tau_{2}\right)-x_{2}^{*}\right)^{2}}{k+x_{1}^{*}} \\
& +\frac{a_{2}\left(x_{2}\left(t+\tau_{2}\right)-x_{2}^{*}\right)\left(x_{2}\left(t+\tau_{2}\right)-x_{2}(t)\right)}{k+x_{1}^{*}} \\
& +\frac{a_{2} x_{2}(t)\left(x_{2}\left(t+\tau_{2}\right)-x_{2}^{*}\right)\left(x_{1}(t)-x_{1}^{*}\right)}{\left(k+x_{1}^{*}\right)\left(k+x_{1}(t)\right)} \\
& +\frac{x_{2}^{*} \sigma_{21}^{2} \sigma_{22}^{2}}{2}+\int_{\mathbb{V}}\left[\gamma_{2}(u)-\ln \left(1+\gamma_{2}(u)\right)\right] \lambda \mathrm{d} u .
\end{aligned}
$$

If $\int_{\mathbb{Y}}\left[\gamma_{2}(u)-\ln \left(1+\gamma_{2}(u)\right)\right] \lambda \mathrm{d} u \leq \tilde{\gamma}_{2}$ and $\tilde{\gamma}_{2}$ are positive constants and $\sigma_{j 2}>0(j=1,2)$ are sufficiently small, then

$$
\begin{aligned}
\mathscr{L} W_{21} \leq & -\frac{a_{2}\left(x_{2}\left(t+\tau_{2}\right)-x_{2}^{*}\right)^{2}}{k+x_{1}^{*}} \\
& +\frac{a_{2} x_{2}^{2}\left(t+\tau_{2}\right)+x_{2}(t) x_{2}^{*}}{k+x_{1}^{*}}+\frac{x_{2}^{*} \sigma_{21}^{2} \sigma_{22}^{2}}{2} \\
& +\frac{x_{2}(t)\left[x_{1}(t) x_{2}\left(t+\tau_{2}\right)-x_{2}^{*} x_{1}(t)-x_{1}^{*} x_{2}\left(t+\tau_{2}\right)+x_{1}^{*} x_{2}^{*}\right]}{\left(k+x_{1}^{*}\right)\left(k+x_{1}(t)\right)} \\
& +\int_{\mathbb{V}}\left[\gamma_{2}(u)-\ln \left(1+\gamma_{2}(u)\right)\right] \lambda \mathrm{d} u \\
\leq & -\frac{a_{2}\left(x_{2}\left(t+\tau_{2}\right)-x_{2}^{*}\right)^{2}}{k+x_{1}^{*}} \\
& +\frac{P_{2}\left[\left(a_{2} P_{2}+x_{2}^{*}\right)\left(k+P_{1}\right)+P_{1} P_{2}+x_{1}^{*} x_{2}^{*}\right]}{\left(k+x_{1}^{*}\right)\left(k+Q_{1}\right)} \\
& +\frac{x_{2}^{*} \sigma_{21}^{2} \sigma_{22}^{2}}{2}+\tilde{\gamma}_{2} .
\end{aligned}
$$

Based on (C.16), $W_{22}(t)$ is defined as follows,

$$
W_{22}(t)=W_{21}(t)+\frac{a_{2}}{\left(k+x_{1}^{*}\right)} \int_{t}^{t+\tau_{2}}\left(x_{2}(s)-x_{2}^{*}\right)^{2} \mathrm{~d} s .
$$

By using simple computations, it can be obtained that

$$
\begin{aligned}
\mathscr{L} W_{22} \leq & -\frac{a_{2}\left(x_{2}(t)-x_{2}^{*}\right)^{2}}{k+x_{1}^{*}} \\
& +\frac{P_{2}\left[\left(a_{2} P_{2}+x_{2}^{*}\right)\left(k+P_{1}\right)+P_{1} P_{2}+x_{1}^{*} x_{2}^{*}\right]}{\left(k+x_{1}^{*}\right)\left(k+Q_{1}\right)} \\
& +\frac{x_{2}^{*} \sigma_{21}^{2} \sigma_{22}^{2}}{2}+\tilde{\gamma}_{2},
\end{aligned}
$$

where $P_{1}, P_{2}, Q_{1}$ have been defined in Lemma 2 .

Fourthly, we construct the following function

$$
W_{23}(t)=\frac{\left[x_{2}\left(t+\tau_{2}\right)-x_{2}^{*}\right]^{2}}{2}
$$

By using similar arguments in (C.16) and (C.18), it follows from simple computations that

$$
\begin{aligned}
\mathscr{L} W_{23}= & a_{2} x_{2}\left(t+\tau_{2}\right)\left(x_{2}\left(t+\tau_{2}\right)-x_{2}^{*}\right)\left(\frac{x_{2}^{*}}{k+x_{1}^{*}}-\frac{x_{2}(t)}{k+x_{1}(t)}\right) \\
& +\sigma_{21}^{2} x_{2}^{* 2}+\frac{\sigma_{22}^{2}}{2}+\int_{\mathbb{Y}}\left[\gamma_{2}(u)-\ln \left(1+\gamma_{2}(u)\right)\right] \lambda \mathrm{d} u \\
= & a_{2} x_{2}\left(t+\tau_{2}\right)\left(x_{2}\left(t+\tau_{2}\right)-x_{2}^{*}\right) \\
& \cdot\left[\frac{k\left(x_{2}^{*}-x_{2}\left(t+\tau_{2}\right)+x_{2}\left(t+\tau_{2}\right)-x_{2}(t)\right)}{\left(k+x_{1}^{*}\right)\left(k+x_{1}(t)\right)}\right. \\
& +\frac{\left.x_{2}^{*} x_{1}(t)-x_{1}^{*} x_{2}(t)\right]+\sigma_{21}^{2} x_{2}^{* 2}+\frac{\sigma_{22}^{2}}{2}}{\left.\left(k+x_{1}^{*}\right)\left(k+x_{1}(t)\right)\right]} \\
& +\int_{\mathbb{Y}}\left[\gamma_{2}(u)-\ln \left(1+\gamma_{2}(u)\right)\right] \lambda \mathrm{d} u \\
\leq & -a_{2} k x_{2}\left(t+\tau_{2}\right) \frac{\left(x_{2}\left(t+\tau_{2}\right)-x_{2}^{*}\right)^{2}}{\left(k+x_{1}^{*}\right)\left(k+x_{1}(t)\right)} \\
& +a_{2} x_{2}^{2}\left(t+\tau_{2}\right) \frac{k x_{2}\left(t+\tau_{2}\right)+x_{2}^{*} x_{1}(t)}{\left(k+x_{1}^{*}\right)\left(k+x_{1}(t)\right)} \\
& +a_{2} x_{2}\left(t+\tau_{2}\right) \frac{x_{2}^{*} x_{2}(t)\left(k x_{2}\left(t+\tau_{2}\right)+x_{2}(t)\right)}{\left(k+x_{1}^{*}\right)\left(k+x_{1}(t)\right)} \\
& +\sigma_{21}^{2} x_{2}^{* 2}+\frac{\sigma_{22}^{2}}{2}+\int_{\mathbb{Y}}\left[\gamma_{2}(u)-\ln \left(1+\gamma_{2}(u)\right)\right] \lambda \mathrm{d} u .
\end{aligned}
$$

If $\int_{\mathbb{Y}}\left[\gamma_{2}(u)-\ln \left(1+\gamma_{2}(u)\right)\right] \mathrm{d} u \leq \tilde{\gamma}_{2}$ and $\tilde{\gamma}_{2}$ are positive constants and $\sigma_{j 2}>0(j=1,2)$ is sufficiently small, then

$$
\begin{aligned}
\mathscr{L} W_{23} \leq & -a_{2} k P_{2} \frac{\left(x_{2}\left(t+\tau_{2}\right)-x_{2}^{*}\right)^{2}}{\left(k+x_{1}^{*}\right)\left(k+P_{1}\right)} \\
& +\frac{a_{2} P_{2}^{2}\left[k P_{2}\left(1+x_{2}^{*}\right)+x_{2}^{*}\left(P_{1}+P_{2}\right)\right]}{\left(k+x_{1}^{*}\right)\left(k+Q_{1}\right)}+\sigma_{21}^{2} x_{2}^{* 2}+\frac{\sigma_{22}^{2}}{2} \\
& +\int_{\mathbb{Y}}\left[\gamma_{2}(u)-\ln \left(1+\gamma_{2}(u)\right)\right] \lambda \mathrm{d} u \\
\leq & -a_{2} k P_{2} \frac{\left(x_{2}\left(t+\tau_{2}\right)-x_{2}^{*}\right)^{2}}{\left(k+x_{1}^{*}\right)\left(k+P_{1}\right)} \\
& +\frac{a_{2} P_{2}^{2}\left[k P_{2}\left(1+x_{2}^{*}\right)+x_{2}^{*}\left(P_{1}+P_{2}\right)\right]}{\left(k+x_{1}^{*}\right)\left(k+Q_{1}\right)} \\
& +\sigma_{21}^{2} x_{2}^{* 2}+\frac{\sigma_{22}^{2}}{2}+\tilde{\gamma}_{2},
\end{aligned}
$$

where $P_{1}, P_{2}, Q_{1}$ have been defined in Lemma 2 .

According to (C.21), $W_{24}(t)$ is defined as follows:

$$
W_{24}(t)=W_{23}+\int_{t}^{t+\tau_{2}} a_{2} k P_{2} \frac{\left(x_{2}(s)-x_{2}^{*}\right)^{2}}{\left(k+x_{1}^{*}\right)\left(k+P_{1}\right)} \mathrm{d} s .
$$

It follows from simple computations that 


$$
\begin{aligned}
\mathscr{L} W_{24} \leq & -a_{2} k P_{2} \frac{\left(x_{2}(t)-x_{2}^{*}\right)^{2}}{\left(k+x_{1}^{*}\right)\left(k+P_{1}\right)} \\
& +\frac{a_{2} P_{2}^{2}\left[k P_{2}\left(1+x_{2}^{*}\right)+x_{2}^{*}\left(P_{1}+P_{2}\right)\right]}{\left(k+x_{1}^{*}\right)\left(k+Q_{1}\right)} \\
& +\sigma_{21}^{2} x_{2}^{* 2}+\frac{\sigma_{22}^{2}}{2}+\tilde{\gamma}_{2} .
\end{aligned}
$$

Let $W_{2}(t)=W_{22}(t)+W_{24}(t)$; it follows from (C.18) and (C.23) that

$$
\begin{aligned}
\mathscr{L} W_{2}= & -\frac{a_{2}\left[k\left(1+Q_{2}\right)+Q_{1}\right]}{\left(k+x_{1}^{*}\right)\left(k+P_{1}\right)}\left(x_{2}(t)-x_{2}^{*}\right)^{2} \\
& +\frac{a_{2} P_{2}^{2}\left[k P_{2}\left(1+x_{2}^{*}\right)+x_{2}^{*}\left(P_{1}+P_{2}\right)\right]}{\left(k+x_{1}^{*}\right)\left(k+Q_{1}\right)} \\
& +\sigma_{21}^{2} x_{2}^{* 2}+\frac{\sigma_{22}^{2}}{2}+\frac{x_{2}^{*} \sigma_{21}^{2} \sigma_{22}^{2}}{2}+2 \tilde{\gamma}_{2} .
\end{aligned}
$$

By integrating both sides of (C.24) from 0 to $t$ and deriving expectation, it gives that

$$
\begin{aligned}
\mathbb{E} W_{2}(t)-\mathbb{E} W_{2}(0) \leq- & \frac{a_{2}\left[k\left(1+Q_{2}\right)+Q_{1}\right]}{\left(k+x_{1}^{*}\right)\left(k+P_{1}\right)} \mathbb{E} \int_{0}^{t}\left(x_{2}(s)-x_{2}^{*}\right)^{2} \mathrm{~d} s \\
+ & \left\{\frac{P_{2}\left[\left(a_{2} P_{2}+x_{2}^{*}\right)\left(k+P_{1}\right)+P_{1} P_{2}+x_{1}^{*} x_{2}^{*}\right]}{\left(k+x_{1}^{*}\right)\left(k+Q_{1}\right)}\right. \\
& +\frac{a_{2} P_{2}^{2}\left[k P_{2}\left(1+x_{2}^{*}\right)+x_{2}^{*}\left(P_{1}+P_{2}\right)\right]}{\left(k+x_{1}^{*}\right)\left(k+Q_{1}\right)} \\
& \left.+\sigma_{21}^{2} x_{2}^{* 2}+\frac{\sigma_{22}^{2}}{2}+\frac{x_{2}^{*} \sigma_{21}^{2} \sigma_{22}^{2}}{2}+2 \tilde{\gamma}_{2}\right\} t .
\end{aligned}
$$

It follows from (C.25) that

$$
\limsup _{t \rightarrow \infty} \frac{1}{t} \mathbb{E} \int_{0}^{t}\left[x_{2}(s)-x_{2}^{*}\right]^{2} \mathrm{~d} s \leq B_{2}
$$

where $B_{2}$ is defined as follows:

$$
B_{2}=\frac{P_{2}\left(k+P_{1}\right)\left\{\left[\left(a_{2} P_{2}+x_{2}^{*}\right)\left(k+P_{1}\right)+P_{1} P_{2}+x_{1}^{*} x_{2}^{*}\right]+a_{2} P_{2}\left[k P_{2}\left(1+x_{2}^{*}\right)+x_{2}^{*}\left(P_{1}+P_{2}\right)\right]\right\}}{a_{2}\left(k+Q_{1}\right)\left[k\left(1+Q_{2}\right)+Q_{1}\right]}+\frac{x_{2}^{*} \sigma_{21}^{2}\left(\sigma_{22}^{2}+2 x_{2}^{*}\right)+\sigma_{22}^{2}+4 \tilde{\gamma}_{2}}{2 a_{2}\left[k\left(1+Q_{2}\right)+Q_{1}\right]}
$$

\section{Conflicts of Interest}

All authors of this article declare that there is no conflict of interests regarding the publication of this article. They have no proprietary, financial, professional, or other personal interest of any nature or kind in any product, service, and/or company that could be construed as influencing the position presented in or review of this article.

\section{Acknowledgments}

This work is supported by the National Natural Science Foundation of China (Grant no. 61673099), Hebei Province Natural Science Foundation (Grant no. F2015501047), and Fundamental Research Funds for the Central Universities of Education Ministry (Grant no. N162304006).

\section{References}

[1] H. R. Thieme, Mathematics in Population Biology, Princeton University Press, Princeton, NJ, USA, 2003.

[2] T. Das, R. N. Mukherjee, and K. S. Chaudhuri, "Bioeconomic harvesting of a prey predator fishery," Journal of Biological Dynamics, vol. 3, no. 5, pp. 447-462, 2009.

[3] K. Chakraborty, S. Jana, and T. K. Kar, "Global dynamics and bifurcation in a stage structured prey predator fishery model with harvesting," Applied Mathematics and Computation, vol. 218, no. 18, pp. 9271-9290, 2012.

[4] G. I. Bischi, F. Lamantia, and D. Radi, "A prey predator fishery model with endogenous switching of harvesting strategy,"
Applied Mathematics and Computation, vol. 219, no. 20, pp. 10123-10142, 2013.

[5] X. Zhang and H. Zhao, "Stability and bifurcation of a reactiondiffusion predator-prey model with non-local delay and Michaelis-Menten-type prey-harvesting," International Journal of Computer Mathematics, vol. 93, no. 9, pp. 1447-1469, 2015.

[6] S. Lenhart and J. T. Workman, Optimal Control Applied to Biological Models, Chapman and Hall/CRC Mathematical and Computational Biology, Chapman and Hall/CRC, Boca Raton, FL, USA, 2007.

[7] Y. Xue, S. Tang, and J. Liang, "Optimal timing of interventions in fishery resource and pest management," Nonlinear Analysis: Real World Applications, vol. 13, no. 4, pp. 1630-1646, 2012.

[8] S. M. Bouguima and S. Benzerdjeb, "An age-structured fishery model: dynamics and optimal management with perfect elastic demand," Applied Mathematical Modelling, vol. 40, no. 1, pp. 218-232, 2016.

[9] Y. Kuang, Delay Differential Equations with Applications in Population Dynamics, Academic Press, Boston, MA, USA, 1993.

[10] A. F. Nindjin, M. A. Aziz-Alaoui, and M. Cadivel, "Analysis of a predator prey model with modified Leslie-Gower and Holling type II schemes with time delay," Nonlinear Analysis: Real World Applications, vol. 7, no. 5, pp. 1104-1118, 2006.

[11] H. Zhao, X. Zhang, and X. Huang, "Hopf bifurcation and spatial patterns of a delayed biological economic system with diffusion," Applied Mathematics and Computation, vol. 266, pp. 462-480, 2015.

[12] C. Liu, N. Lu, and Q. Zhang, "Dynamical analysis in a hybrid bioeconomic system with multiple time delays and strong 
Allee effect," Mathematics and Computers in Simulation, vol. 136, pp. 104-131, 2017.

[13] T. K. Kar and A. Ghorai, "Dynamic behaviour of a delayed predator prey model with harvesting," Applied Mathematics and Computation, vol. 217, no. 22, pp. 9085-9104, 2011.

[14] G. Zhang, Y. Shen, and B. Chen, "Positive periodic solutions in a non-selective harvesting predator-prey model with multiple delays," Journal of Mathematical Analysis and Applications, vol. 395, no. 1, pp. 298-306, 2012.

[15] G. Zhang, Y. Shen, and B. Chen, "Hopf bifurcation of a predator-prey system with predator harvesting and two delays," Nonlinear Dynamics, vol. 73, no. 4, pp. 2119-2131, 2013.

[16] C. Liu, Q. Zhang, J. Li, and W. Yue, "Stability analysis in a delayed prey predator resource model with harvest effort and stage structure," Applied Mathematics and Computation, vol. 238, no. 1, pp. 177-192, 2014.

[17] D. Pal and G. S. Mahapatra, "A bioeconomic modeling of twoprey and one-predator fishery model with optimal harvesting policy through hybridization approach," Applied Mathematics and Computation, vol. 242, pp. 748-763, 2014.

[18] R. Yuan, W. Jiang, and Y. Wang, "Saddle-node-Hopf bifurcation in a modified Leslie-Gower predator-prey model with time-delay and prey harvesting," Journal of Mathematical Analysis and Applications, vol. 422, no. 2, pp. 1072-1090, 2015.

[19] M. Liu, "Optimal harvesting policy of a stochastic predator prey model with time delay," Applied Mathematics Letters, vol. 48, pp. 102-108, 2015.

[20] Q. Liu, D. Jiang, N. Shi, T. Hayat, and A. Alsaedi, "Asymptotic behavior of a stochastic delayed SEIR epidemic model with nonlinear incidence," Physica A: Statistical Mechanics and its Applications, vol. 462, pp. 870-882, 2016.

[21] Q. Liu, D. Jiang, N. Shi, T. Hayat, and A. Alsaedi, "Asymptotic behaviors of a stochastic delayed SIR epidemic model with nonlinear incidence," Communications in Nonlinear Science and Numerical Simulation, vol. 40, pp. 89-99, 2016.

[22] L. Liu, X. Meng, and T. Zhang, "Optimal control strategy for an impulsive stochastic competition system with time delays and jumps," Physica A: Statistical Mechanics and its Applications, vol. 477, pp. 99-113, 2017.

[23] H. Qiu and W. Deng, "Optimal harvesting of a stochastic delay competitive Lotka Volterra model with Lévy jumps," Applied Mathematics and Computation, vol. 317, pp. 210-222, 2018.

[24] J. Bao and C. Yuan, "Stochastic population dynamics driven by Lévy noise," Journal of Mathematical Analysis and Applications, vol. 391, no. 2, pp. 363-375, 2012.

[25] M. Liu and K. Wang, "Stochastic Lotka Volterra systems with Lévy noise," Journal of Mathematical Analysis and Applications, vol. 410, no. 2, pp. 750-763, 2014.

[26] H. S. Gordon, "The economic theory of a common property resource: the fishery," Journal of Political Economy, vol. 62, no. 2, pp. 124-142, 1954.

[27] Y. Zhang, Q. Zhang, and X. G. Yan, "Complex dynamics in a singular Leslie Gower predator prey bioeconomic model with time delay and stochastic fluctuations," Physica A: Statistical Mechanics and its Applications, vol. 404, pp. 180-191, 2014.

[28] X. Meng and Q. Zhang, "Complex dynamics in a singular delayed bioeconomic model with and without stochastic fluctuation," Discrete Dynamics in Nature and Society, vol. 2015, Article ID 302494, 15 pages, 2015.
[29] Y. Zhang, Y. Zheng, X. Liu, Q. Zhang, and A. Li, "Dynamical analysis of a differential algebraic bio-economic model with stage-structured and stochastic fluctuations," Physica A: Statistical Mechanics and its Applications, vol. 462, pp. 222-229, 2016.

[30] C. Liu, L. Yu, Q. Zhang, and Y. Li, "Dynamic analysis of a hybrid bioeconomic plankton system with double time delays and stochastic fluctuations," Applied Mathematics and Computation, vol. 316, pp. 115-137, 2018.

[31] P. Feng, "Analysis of a delayed predator-prey model with ratio-dependent functional response and quadratic harvesting," Journal of Applied Mathematics and Computing, vol. 44, no. 1-2, pp. 251-262, 2014.

[32] C. Liu, N. Lu, Q. Zhang, J. Li, and P. Liu, "Modeling and analysis in a prey-predator system with commercial harvesting and double time delays," Applied Mathematics and Computation, vol. 281, pp. 77-101, 2016.

[33] X. Yang, L. Chen, and J. Chen, "Permanence and positive periodic solution for the single-species nonautonomous delay diffusive models," Computers \& Mathematics with Applications, vol. 32, no. 4, pp. 109-116, 1996.

[34] X. Song and L. Chen, "Optimal harvesting and stability for a two-species competitive system with stage structure," Mathematical Biosciences, vol. 170, no. 2, pp. 173-186, 2001.

[35] J. K. Hale, Theory of Functional Differential Equations, Springer, New York, NY, USA, 1997.

[36] W. Q. Zhu, Nonlinear Dynamics and Control Hamilton Theoretical System Frame, Science Press, Beijing, China, 2003.

[37] D. Huang, H. Wang, J. Feng, and Z. W. Zhu, "Hopf bifurcation of the stochastic model on HAB nonlinear stochastic dynamics," Chaos, Solitons and Fractals, vol. 27, no. 4, pp. 1072-1079, 2006.

[38] X. Mao, Stochastic Differential Equations and Applications, Hardwood Publishing, Chichester, UK, 1997. 


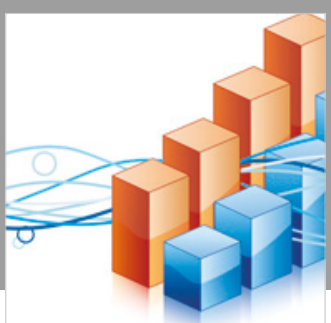

Advances in

Operations Research

\section{-n-m}
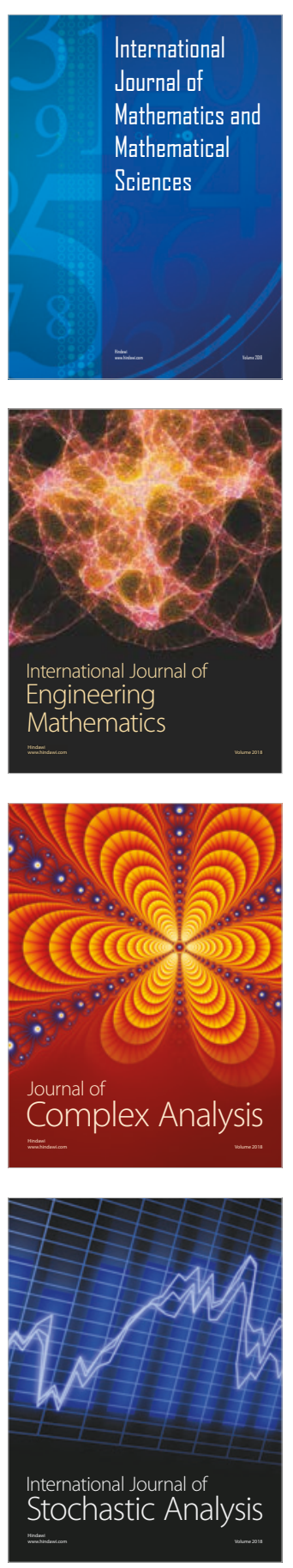
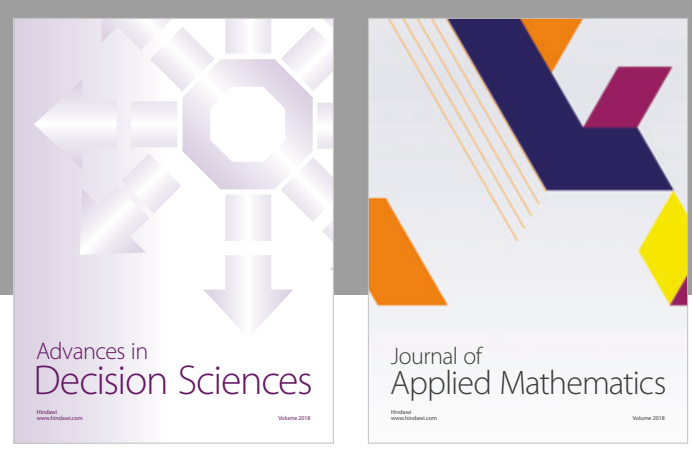

Journal of

Applied Mathematics
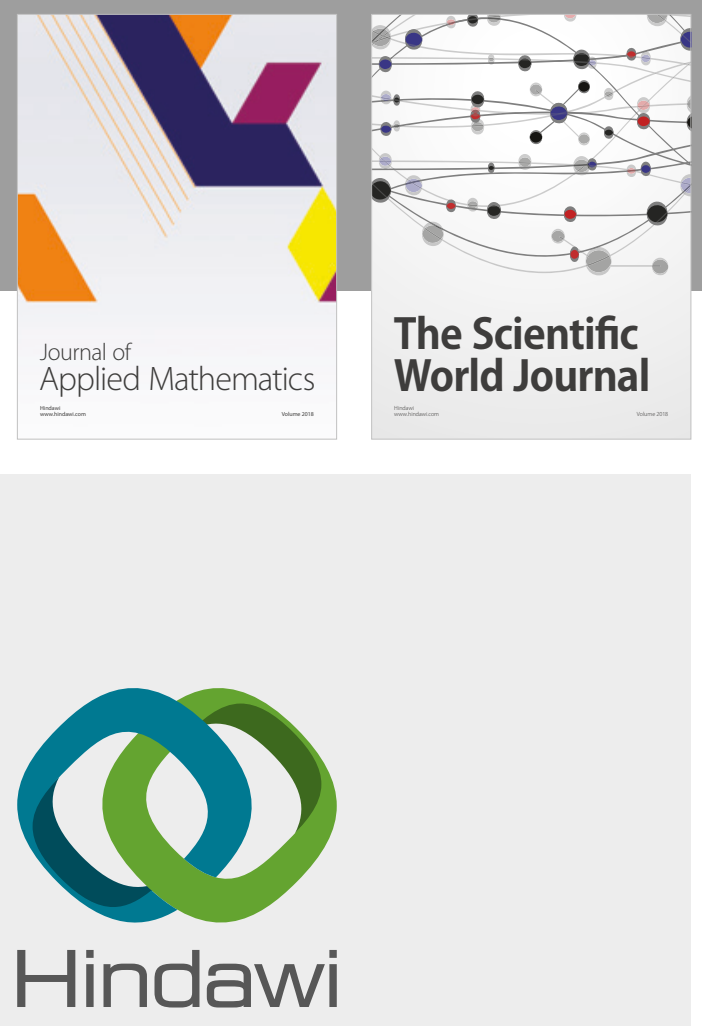

Submit your manuscripts at

www.hindawi.com

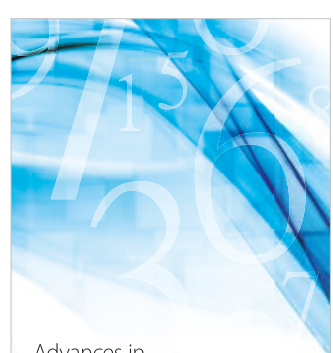

Advances in
Numerical Analysis
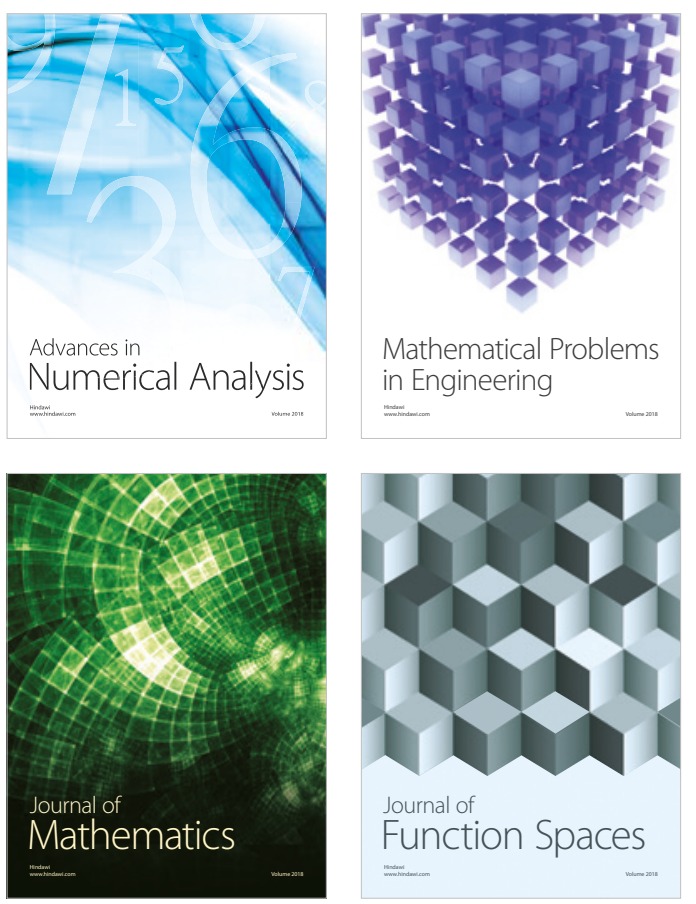

Mathematical Problems in Engineering

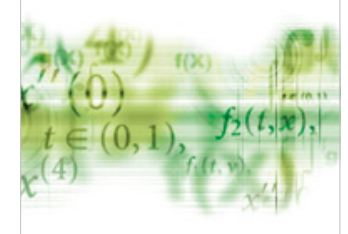

International Journal of

Differential Equations

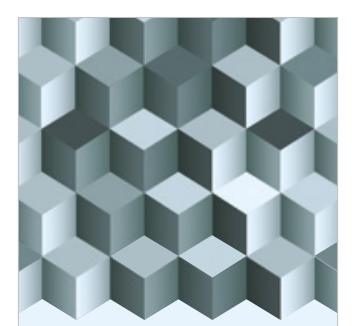

Journal of

Function Spaces
The Scientific

World Journal

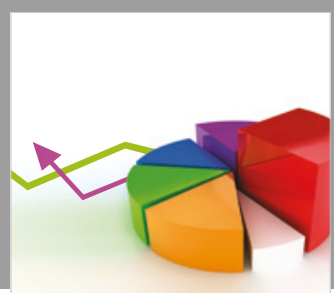

Journal of

Probability and Statistics
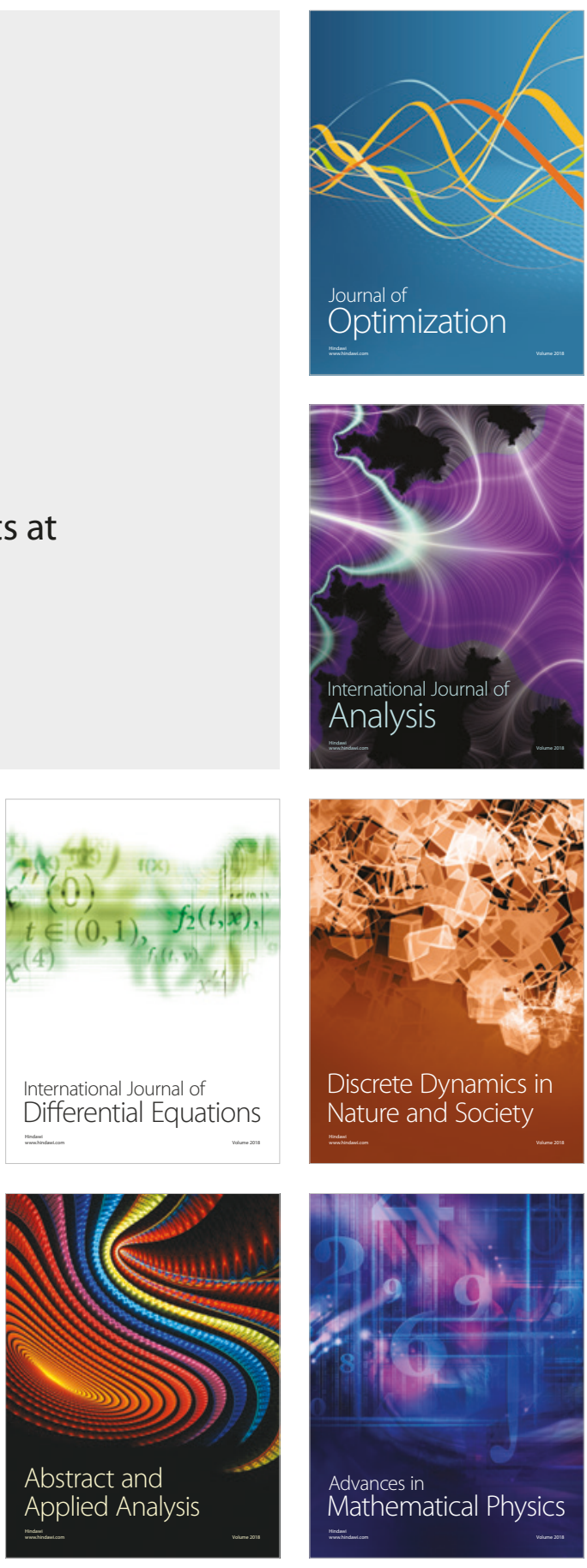